\title{
1 Transcript- and annotation-guided genome assembly of the European starling
}

2

3 Katarina C. Stuart ${ }^{1 \dagger}$, Richard J. Edwards ${ }^{2 \dagger}$, Yuanyuan Cheng ${ }^{3}$, Wesley C. Warren ${ }^{4}$, David W.

4 Burt $^{5}$, William B. Sherwin ${ }^{1}$, Natalie R. Hofmeister ${ }^{6,7}$, Scott J. Werner ${ }^{8}$, Gregory F. Ball ${ }^{9}$,

Melissa Bateson ${ }^{10}$, Matthew C. Brandley ${ }^{11}$, Katherine L. Buchanan ${ }^{12}$, Phillip Cassey ${ }^{13}$, David F. Clayton ${ }^{14}$, Tim De Meyer ${ }^{15}$, Simone L. Meddle ${ }^{16}$, Lee A. Rollins ${ }^{1}$

${ }^{1}$ Evolution \& Ecology Research Centre, School of Biological, Earth and Environmental Sciences, UNSW Sydney, Sydney, New South Wales, Australia

${ }^{2}$ School of Biotechnology and Biomolecular Sciences, UNSW Sydney, Sydney, New South Wales, Australia

${ }^{3}$ School of Life and Environmental Sciences, The University of Sydney, Sydney, New South Wales, Australia

${ }^{4}$ Department of Animal Sciences, Institute for Data Science and Informatics, The University of Missouri, Columbia, Missouri, USA

${ }^{5}$ Office of the Deputy Vice-Chancellor (Research and Innovation), The University of Queensland, Brisbane, Australia

${ }^{6}$ Department of Ecology and Evolutionary Biology, Cornell University, Ithaca, NY 14850

${ }^{7}$ Fuller Evolutionary Biology Program, Cornell Lab of Ornithology, Ithaca, NY 14850

${ }^{8}$ United States Department of Agriculture, Animal and Plant Health Inspection Service, Wildlife Services, National Wildlife Research Center, Fort Collins, Colorado, USA.

${ }^{9}$ Department of Psychology, University of Maryland, College Park, MD 20742 USA

${ }^{10}$ Institute of Neuroscience, Newcastle University, Newcastle upon Tyne, UK

${ }^{11}$ Carnegie Museum of Natural History, Pittsburgh, Pennsylvania, USA

${ }^{12}$ School of Life and Environmental Sciences, Deakin University, Waurn Ponds, VIC, 3228, Australia

${ }^{13}$ Invasion Science \& Wildlife Ecology Lab, University of Adelaide, Adelaide SA 5005, Australia

${ }^{14}$ Department of Genetics \& Biochemistry, Clemson University, South Carolina 29634 ${ }^{15}$ Dept. of Data Analysis \& Mathematical Modelling, Faculty of Bioscience Engineering, Ghent University, Ghent, Belgium 16 The Roslin Institute, The Royal (Dick) School of Veterinary Studies, The University of Edinburgh, Midlothian, EH25 9RG, UK 
$\dagger$ Joint first authors

\section{Abstract (250 words)}

The European starling, Sturnus vulgaris, is an ecologically significant, globally invasive avian species that is also suffering from a major decline in its native range. Here, we present the genome assembly and long-read transcriptome of an Australian-sourced European starling $(S$. vulgaris vAU), and a second North American genome (S. vulgaris vNA), as complementary reference genomes for population genetic and evolutionary characterisation. S. vulgaris vAU combined 10x Genomics linked-reads, low-coverage Nanopore sequencing, and PacBio IsoSeq full-length transcript scaffolding to generate a $1050 \mathrm{Mb}$ assembly on 1,628 scaffolds (72.5 Mb scaffold N50). Species-specific transcript mapping and gene annotation revealed high structural and functional completeness (94.6\% BUSCO completeness). Further scaffolding against the high-quality zebra finch (Taeniopygia guttata) genome assigned $98.6 \%$ of the assembly to 32 putative nuclear chromosome scaffolds. Rapid, recent advances in sequencing technologies and bioinformatics software have highlighted the need for evidence-based assessment of assembly decisions on a case-by-case basis. Using S. vulgaris vAU, we demonstrate how the multifunctional use of PacBio Iso-Seq transcript data and complementary homology-based annotation of sequential assembly steps (assessed using a new tool, SAAGA) can be used to assess, inform, and validate assembly workflow decisions. We also highlight some counter-intuitive behaviour in traditional BUSCO metrics, and present BUSCOMP, a complementary tool for assembly comparison designed to be robust to differences in assembly size and base-calling quality. Finally, we present a second starling assembly, S. vulgaris vNA, to facilitate comparative analysis and global genomic research on this ecologically important species. 
Keywords: Sturnus vulgaris, genome assembly, genome assessment, genome annotation,

full-length transcripts

Running head: Genome assembly of the European starling

\section{Introduction}

The European starling (Sturnus vulgaris) is a globally invasive passerine that was

Australia, New Zealand, and South Africa during the mid-late $19^{\text {th }}$ century (Feare 1985).

More recently, the species was accidentally introduced into South America (Palacio et al. 2016). Since these introductions the invasive ranges of the starling have been expanding, with the species now occupying a range in excess of $38,400,000 \mathrm{~km}^{2}$ globally (BirdLife International 2020), posing threats to the economics and health of the agriculture industry, as well as local biodiversity (Bomford \& Sinclair 2002; Koch et al., 2009; Palacio et al., 2016; Linz et al., 2017). Recent molecular ecology studies of individuals from the invasive ranges of North America, Australia, and South Africa report that these populations are undergoing rapid and independent evolution in response to novel local selection pressures (Phair et al., 2018; Hofmeister et al., 2019; Bodt et al., 2020; Stuart \& Cardilini et al., 2020), a common phenomenon in many invasive populations (Prentis et al., 2008). This suggests the starling has a flexible invasion strategy, potentially enabling colonisation of ecosystems vastly different from those in their native range.

Despite their invasive range success, European starlings are increasingly of ecological concern within their native range (Rintala et al., 2003; Robinson et al., 2005). High densities of native range starlings have traditionally been supported by cattle farming across Europe, because starlings preferentially feed in open grasslands, and benefit from invertebrates in 
overturned soil produced by livestock grazing (Coleman 1977). A shift towards modern indoor cattle rearing processes across Europe may contribute to the decline in starling numbers, which has been a concern since the 1980s (Wretenberg et al., 2006). This decline is reflected globally, with starling and other avifauna numbers decreasing sharply over the last few decades (Spooner et al., 2018; Rosenberg et al., 2019), though this may be further amplified for starling populations subjected to control strategies to reduce their economic impact (Linz et al., 2007). The biological and ecological importance of this species is evident from its prolific use in research, as it is the most studied non-domesticated passerine (Bateson \& Feenders 2010). It is evident that future research on the European starling will focus on identifying patterns of evolutionary diversification, and investigating genes associated with invasion success. Such research provides important information for the improvement of control measures and may also provide insight into recovery and dispersive potential in other species that would benefit global conservation efforts. For this, a high-quality, annotated reference genome is essential.

Once reliant on large consortia, assembling high-quality reference genomes for genetic analyses is now commonplace. Nevertheless, de novo assembly of non-model organism genomes still poses many challenges. Best practices may have not been established for the study species/data, and basic information such as genome size, repeat landscape, and ploidy may be unknown. Furthermore, high-quality references can be generated in multiple ways, which can serve varied research purposes. Rapid developments in both sequencing technology and bioinformatics methods can quickly outdate benchmarking attempts. Whilst not always documented in final publications, the standard practice for non-model species genomes is to select from multiple assemblies generated using different assembly methods, none of which is universally best (Rhie et al., 2020; Whibley et al., 2020). This complexity 
can be magnified further when sequencing occurs across multiple technology platforms that decisions, based on the quality of the genome assemblies produced.

A multitude of tools and approaches are available for genome assembly assessment, though some may not be applicable or feasibly implemented for a particular species/assembly

111 and/or the data available (e.g. Bradnam et al., 2013; Hunt et al., 2013; Yuan et al., 2017).

112 Common approaches employed to guide genome assembly decisions focus on contiguity

113 (how continuous the assembled sequences are) and completeness (whether the assembly

114 contains all the genetic information for that species). Two such approaches are assembly

115 statistics (e.g., contig/scaffold counts, and L50/N50 statistics of the number and shortest

116 length of sequences needed to cover $50 \%$ of the assembly) and "Benchmarking Universal

117 Single Copy Orthologs" (BUSCO) estimates of genome completeness (Simão et al., 2015).

118 Assembly statistics are very quick to generate and easy to understand, but interpretation can

119 be challenging due to hidden assembly errors and artefacts, which can create false signals.

120 BUSCO assesses the presence or absence of highly conserved lineage-specific genes but is

121 limited to a set of common single-copy genes that may represent easier regions of the genome

122 to sequence and assemble based on current bioinformatics technologies. Furthermore,

123 BUSCO analysis is vulnerable to unpredictable misreporting of presence/completeness for

124 specific genes as a consequence of assembly differences elsewhere in the genome (Edwards et 125 al., 2018; Edwards 2019; Field et al., 2020; Edwards et al., 2021). In addition to the above 126 drawbacks, these methods do not explicitly test the genome assembly's ability to perform the

127 role for which it was intended (e.g., to serve as a reference genome for specific genomic 128 analysis). 
Here, we present the first official release of the European starling draft genome, $S$.

131 transcripts, together with genomic data for this species. In this paper, we complement genome

132 statistics and BUSCO completeness with transcriptome- and annotation-based assessments

133 that help determine genome assembly quality and completeness in the absence of a reference

134 genome to benchmark against. We demonstrate how full-length transcripts can be utilised in

135 genome assembly scaffolding and assessment, in addition to transcriptome construction and

136 annotation. We show how BUSCOMP (https://github.com/slimsuite/buscomp) can help avoid

137 over-interpretation or misinterpretation of small differences in BUSCO completeness. We

138 also explore how lightweight homology-based annotation by GEMoMA (Keilwagen et al.,

139 2018), can be used as an assembly assessment using a new tool, SAAGA

140 (https://github.com/slimsuite/saaga). Finally, we compare the Australian S. vulgaris vAU

141 assembly (GCF_ JAGFZL000000000) with an additional (short read) assembly of a North

142 American bird, S. vulgaris vNA (GCF_001447265.1), enabling reference-specific biases to be

143 identified in future starling genomics studies.

\section{Material and Methods}

\subsection{Genome assembly and scaffolding}

147 The S. vulgaris vAU genome assembly used 10x Chromium linked reads and low coverage

148 ONT long reads (Appendix 1: Genomic DNA sample collection, gDNA extraction, and

149 sequencing), and was produced via eight assembly steps (Fig. 1). The 10x reads were

150 assembled into an initial diploid assembly using SUPERNOVA (v2.1.1) (Weisenfeld et al.,

151 2017) with barcode fraction and reads subsample calculated following SUPERNOVA best

152 practices for a genome size based on k-mer counts calculation by JELLYFISH v2.2.10 (Marçais 
$\&$ Kingsford 2011) (parameters: bcfrac $=0.8$, maxreads $=550$ million, Supplementary

154 Materials: Appendix 2, Validation of SUPERNOVA genome size prediction using JELLYFISH,

155 Supplementary Materials: Fig. S1). This assembly was then split into non-redundant primary

156 and alternative haploid assemblies using DIPLOIDOCUS (parameters: runmode= diphapnr)

157 (v0.9.5) (https://github.com/slimsuite/diploidocus). First, both SUPERNOVA pseudohap2

158 assemblies were combined and any sequences lacking definitive base calls (100\% Ns) were

159 removed. Remaining scaffolds were size-sorted and gaps reduced in size to a maximum of 10

160 Ns then subject to an all-by-all search with MINIMAP2 (v2.17) (Li 2018) (--cs -p 0.0001 -X

161 asm20 -N 250). (Note that gap size reduction is used for MINIMAP2 searching only, and the

162 non-redundant pseudodiploid assembly produced has the same gap sizes as generated by

163 SUPERNOVA.). Any sequences that were $100 \%$ contained within another sequence were

164 removed. Where two or more scaffolds had an $100 \%$ identical sequence, only one was kept.

165 Scaffolds are then matched into haplotig pairs based on their SUPERNOVA names. Where a

166 single haplotig is found, it is assigned as diploid, under the assumption that the two original

167 haplotigs were identical with one removed, and added to the primary assembly. (Note: it is

168 possible that only one parent had this scaffold, e.g., a sex chromosome scaffold or structural

169 variant.). If two haplotigs are identified, the longest is assigned to the primary assembly and

170 the shorter to the alternative assembly. The primary assembly should therefore contain an

171 entire haploid copy of the genome, whilst the alternative assembly contains the subset of

172 scaffolds with heterozygous haplotigs.

173 The primary haploid assembly produced by DIPLOIDOCUS was scaffolded using the

174 filtered ONT reads using the program SSPACE-LONGREAD (v1-1) (Boetzer \& Pirovano

175 2014). The filtered ONT reads were then used to gap-fill the assembly using GAPFINISHER

176 (v1.0) (Kammonen et al., 2019). Clustered high-quality Iso-Seq reads (see section $2.2 \mathrm{cDNA}$ 
analysis) were then used for a secondary round of scaffolding using L_RNA_SCAFFOLDER

178 (Xue et al., 2013). Paired-end 10x linked reads were processed with 10X Genomics LONG

RANGER (v2.2) and mapped onto this scaffolded assembly using BWA mem before error

180 correction of SNPs and indels using PILON (v1.23) (Walker et al., 2014) (parameters: --diploid

181 -fix all settings). To validate the scaffolds, the assembly was analysed using the BREAK10x

182 toolkit in SCAFF10x (v3.1) (https://github.com/wtsi-hpag/Scaff10X). The assembly was

183 further checked for assembly artefacts and contamination using DIPLOIDOCUS (parameters:

184 runmode=purgehaplotig \& runmode=vecscreen (ref); runmode=DipCycle was tested yet

185 discarded due to over-pruning, see Supplementary Materials: Fig. S2) (v0.9.5). Avian species

186 are characterised by distinctive and constrained karyotypes, generally comprised of

187 approximately 10 macrochromosomes and approximately 30 indistinguishable

188 microchromosomes (Griffin et al., 2007; O'Connor et al., 2019), a pattern to which the $S$.

189 vulgaris genome conforms (Calafati \& Capanna 1981). Therefore, we aligned our assembly to

190 the chromosome scale assembly of zebra finch (Taeniopygia guttata) (NCBI=

191 GCF_008822105.2) (Balakrishnan et al., 2010) using SATSUMA2

192 (https://github.com/bioinfologics/satsuma2) to create putative chromosomes assuming

193 orthology. This assembly formed the final updated draft genome we present for the species:

194 Sturnus vulgaris vAU.

195 


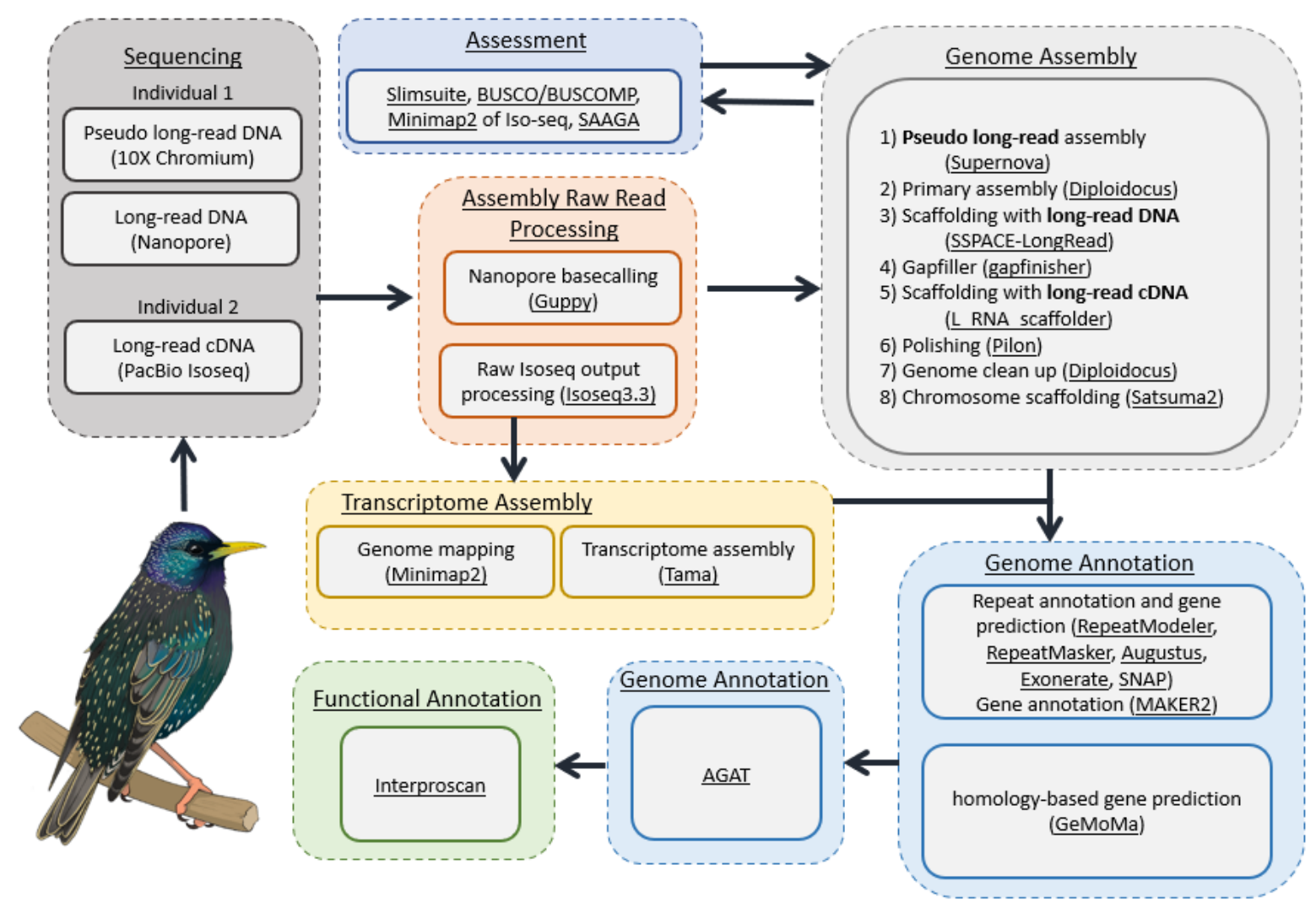

Figure 1: Workflow for genome assembly and annotation. A summary of all the experimental methods used for sequencing, genome assembly, transcriptome assembly, genome annotation, and functional annotation, with programs used underlined.

\subsection{Transcriptome assembly and analysis}

205 Raw PacBio Iso-Seq whole transcript reads (Appendix 3: Transcriptome sample collection,

206 RNA extraction, and sequencing) were processed using the protocol outlined in SMRT LINK

207 (v9.0) (PacBio, California, United States). Briefly, this involved generating Circular

208 Consensus Sequences (CCS) using CCS (v4.2.0), which were then processed using Lima

209 (v1.11.0) for primer removal and demultiplexing. The reads were further processed (PolyA

210 tail minimum length $=8$ ) and clustered using ISO-SEQ (v3.3). The high-quality clustered Iso-

211 Seq reads were then aligned to the reference genome (see section 2.1 Genome assembly and 
scaffolding) using minimap2 (v2.17) (Li 2018), before further processing using TAMA

RNA (Richardson et al., 2017), as well as other available avian Iso-Seq transcriptomes

217 (Workman et al., 2018; Yin et al., 2019).

\subsection{Genome annotation and functional annotation}

219 Each stage of genome assembly was annotated using GEMOMA v1.7.1 (Keilwagen et al.,

2018) using the 26 avian genome annotations available on Ensembl at the time this analysis

was conducted (Supplementary Materials, Table S1) and with the high-quality clustered Iso-

seq, as RNA evidence. The GEMOMA GeMoMaPipeline function was run to complete the full pipeline with a maximum intron size of $200 \mathrm{~kb}$ (parameters: tblastn=false

GeMoMa.m=200000 GeMoMa.Score=ReAlign AnnotationFinalizer.r=SIMPLE pc=true $\mathrm{o}=$ true).

The final S. vulgaris vAU genome assembly was also annotated with MAKER2 (Holt \& Yandell 2011) (BLAST+ v2.9 (Camacho et al., 2009), AUGUSTUS v3.3.2 (Stanke \& Morgenstern 2005), ExONERATE v2.2.0 (Gs \& E 2005), REPEATMASKER v4.0.7 (Smit et al., 2013), REPEATMODELER v1.0.11 (Flynn et al., 2020), and SNAP v0.15.4 (Korf 2004) using repeat-filtered Swiss-Prot protein sequences (downloaded Aug 2018) (UniProt Consortium

\section{2019). A custom AUGUSTUS species database was created by running BUSCO using the}

232 Optimization mode Augustus self-training mode (--long), using the aves database for lineage.

233 MAKER2 was run using the recommended protocol, including generation of a repeat library,

234 and with the TAMA-processed Iso-Seq data included as primary species transcript evidence, 235 and the pre-existing short read liver transcript data (Richardson et al., 2017) provided as 
alternate transcript evidence in the first iteration of the MAKER2 annotation process. We ran

MAKER2 for a total of three training runs, using the hidden Markov models (HMMs) produced

annotation model to produce high-quality and conservative gene predictions. GEMOMA and

MAKER2 annotations for the final S. vulgaris vAU assembly were combined using the AGAT

agat_sp_merge_annotations function to produce the final annotation. Functional annotation

Gene3D, Hamap, ProSiteProfiles, Coils, SMART, CDD, PRINTS, Pro SitePatterns,

SignalP_EUK, Pfam, ProDom, MobiDBLite, PIRSF, TMHMM). BLAST was used to annotate predicted genes using all Swiss-Prot proteins (parameters: -evalue 0.000001 -seg yes -

\subsection{Annotation assessment using SAAGA: Summarise, Annotate \& Assess Genome}

\section{Annotations}

254 predicted proteins to the repeat- and transposase-filter Swiss-Prot protein sequences used for 255 MAKER2 annotation (above). SAAGA performs a reciprocal MMseqs2 (Steinegger \& Söding 256 2017) search of annotated proteins against a (high-quality) reference proteome, identifying 257 best hits for protein identification and employing coverage ratios between query and hit 258 proteins as a means of annotation assessment to generate summary statistics, including: 
- Protein length ratio. The length ratio of the annotated proteins versus its top reference hit

- F1 score. An annotation consistency metric calculated using the formula: protein hit, and REFCOV is the proportion of the best reference protein hit covered by the annotated protein.

- Completeness. The summed percentage coverage of reference proteome.

- Purity. The summed percentage reference coverage of the annotated proteome.

- Homology. The percentage of annotated genes with any hit in reference.

- Orthology. The percentage of annotated genes with reciprocal best hits in reference.

- Duplicity. The mean number of annotated genes sharing the same best reference hit.

- Compression. The number of unique annotated genes that were the top hit for reference proteins, divided by the total number of reference proteins with a hit.

- Multiplicity. The ratio of total number of annotated genes to reference proteins.

274 For protein length ratio and F1 score, values close to 1 means that the query protein closely matches the length of the hit protein, indicating high fidelity of the gene prediction model and underlying assembly. The remaining metrics will be closer to 1 (or 100\%) for complete annotations and assemblies without duplications, akin to BUSCO scores. Although the maximum achievable value for these metrics will generally be unknown, comparative values can be used to assess improvement in assembly and/or annotation.

SAAGA scores may be used to compare alternate annotations of the same assembly, 281 or to compare alternative assemblies in conjunction with consistent annotation. Low genome 282 contiguity, misassembles, or frameshifting indels will affect the quality of predicted genes, 
with poorer assemblies reporting more fragmented or truncated genes. This approach has been program can be run from one line of code and may be parallelised to run much faster than other annotation software (e.g., MAKER2). The ease of this annotation tool opens the way for conducting annotations for the purpose of assessment on sequential or even competing genome annotation steps. Assessing the quality of protein-coding region predictions will help ensure the final genome assembly can produce a high-quality annotation. Here, we used the repeat-filtered Swiss-Prot database used in annotation, and the Gallus gallus reference 292 proteome (UP000000539_9031), to assess predicted protein quality and annotated proteome 293 completeness.

\subsection{Genome assembly completeness assessment}

Assembly contiguity and completeness was assessed for sequential genome assembly steps of the $S$. vulgaris vAU assembly and compared to existing passerine chromosome level assemblies available on NCBI, including the $S$. vulgaris vNA assembly (Assembly accession

298 GCF_001447265.1, Supplementary Material: Appendix 4, Assembly and annotation of the $S$. vulgaris vNA genome version).

\subsubsection{BUSCO and BUSCOMP assembly completeness assessment}

302 lineage). BUSCO resulted were collated across all assemblies using BUSCOMP v0.10.1

303 (https://github.com/slimsuite/buscomp). BUSCOMP collated BUSCO outputs across all

304 genome assembly stages and compiled a maximal non-redundant set of 4727 complete

305 BUSCOs found at single copy in at least one assembly. Compiled BUSCO predicted gene

306 sequences were mapped onto each assembly to be rated with MINIMAP2 V2.17 (Li 2018) and 
re-scored in terms of completeness, thereby providing a robust and consistent means of assessing comparable completeness across assemblies of the same genome.

\subsubsection{PacBio Iso-Seq completeness assessment}

The PacBio Iso-Seq reads were mapped on to genome assemblies using MINIMAP2

311 (parameters: -ax splice -uf --secondary=no --splice-flank=no -C5 -O6,24 -B4) (Li 2018) and

312 the number of Iso-Seq transcripts mapping on to each assembly, and their corresponding mapping quality, was calculated.

\subsubsection{KAT k-mer completeness assessment}

The final genome assembly completeness was assessed by examining the read k-mer frequency distribution with different assembly copy numbers based on the 10x Chromium

317 linked reads using K-MER ANALYsis TOOLKIT (KAT) v2.4.2 (Mapleson et al., 2017) (30 bp

318 trimmed for $\mathrm{R} 1$ reads, and $16 \mathrm{bp}$ trimmed for $\mathrm{R} 2$ reads).

\subsection{Additional genome statistics}

The Iso-Seq and final annotation transcript density, final annotation gene density,

321 global SNP variant density (based on a whole genome data set of 24 individuals from United

322 Kingdom, North America, and Australia, N=8 (Hofmeister et al. 2021), and GC-content were

323 calculated in sliding windows of width $1 \mathrm{Mb}$ using BEDTOOLS v 2.27.1 (Quinlan \& Hall

324 2010), and plotted across the largest 32 scaffolds in our final genome assembly (representing 325 more than $98 \%$ of the total assembly captured on putative chromosomes orthologous to other 326 avian chromosomes) using CIRCLIZE (v 0.4.9) (Gu et al., 2014).

\section{$327 \quad 2.7$ Genome assembly correction}

NCBI VecScreen flagged possible bacterial and adapter contamination in the final $S$.

329 vulgaris $v A U$ assembly, which was missed by earlier contamination screening steps. An 330 updated version of DIPLOIDOCUS (runmode vecscreen) was run to mask shorter adapter 
sequences and flag additional organism contaminates (screenmode=purge vecmask=27). Four

340 trimmed to remove the contaminating sequence (seq 4 trimmed, seq 12 and 31 split into

341 chromosome and unplaced scaffold). Finally, gaps of unknown size were standardised to 100

This paper primarily analyses S. vulgaris vAU1.0 (which we refer to has $S$. vulgaris vAU), while the final NCBI release (accession = JAGFZL000000000) is explicitly referred to as $S$.

346 vulgaris vAU1.1 when relevant.

\subsection{BUSCO versus BUSCOMP performance benchmarking}

BUSCO-containing scaffolds from the DIPLOIDOCUS primary haploid SUPERNOVA

350 for additional BUSCO and BUSCOMP benchmarking (Supplementary Materials: Fig. S3).

351 BUSCO v3.0.2b (Simão et al., 2015) (HMMER v3.2.1 (Wheeler \& Eddy 2013), AUGUSTUS

352 v3.3.2 (Stanke \& Morgenstern 2005), BLAST+ v2.2.31 blast(Camacho et al., 2009),

353 EMBOSS v6.6.0 (Rice et al., 2000)) was run in genome mode with the aves_odb9 dataset

$354(\mathrm{n}=4915)$ on: the non-redundant pseudodiploid ('dipnr'), primary ('pri') and alternative ('alt') 
assemblies; BUSCO-containing scaffolds from the primary assembly ('pribusco'); a reverse'shuffle3'), added in combination to `pribusco` to generate datasets of increasing assembly

360 size without increasing duplication levels ('2n', '3n' and ' $4 \mathrm{n}$ '); ten straight repeats of the ‘pribusco` run (`rep0` to `rep9`). All BUSCO results were processed with BUSCOMP v0.11.0

(MINIMAP2 v2.17). In addition to the full BUSCOMP analysis of all runs, the following subsets were grouped for analysis (Supplementary File 1, BUSCO v3 BUSCOMP output):

- Pseudodip: 'dipnr', 'pri' and 'alt'. (Haploid versus diploid assemblies.)

- Core: 'dipnr', 'pri', 'alt', 'pribusco' and 'revcomp'. (Assembly filtering and manipulation.)

- Duplication: 'copy', 'duplicate', 'triplicate'. (Duplicating scaffolds.)

- Size: 'shuffle1', 'shuffle2', 'shuffle3', '2n', ‘3n', ‘4n'. (Increasing assembly size without duplication.)

- Replicates: 'rep0' to 'rep9'.

The same analysis was repeated with BUSCO v5.0.0 (Simão et al., 2015) (SEPP v4.3.10 (Mirarab et al., 2012), BLAST v2.11.0 (Camacho et al., 2009), HMMer v3.3 (Wheeler \& Eddy 2013), AUGUSTUS v3.3.2 (Stanke \& Morgenstern 2005), Prodigal v2.6.3 (Hyatt et al., 2010), METAEUK v20200908 (Levy Karin et al., 2020)) and the aves_odb10 dataset $(n=8338)$.

\section{Results}

\subsection{Sturnus vulgaris vAU genome assembly}


397 (NUMTs) were located in S. vulgaris vAU1.1, with scaffold 31 (corresponding to the Z 398 chromosome) containing the highest amount (Table S2).

399 Table 1: Summary of sequencing data for Sturnus vulgaris vAU genome assembly and 400 annotation

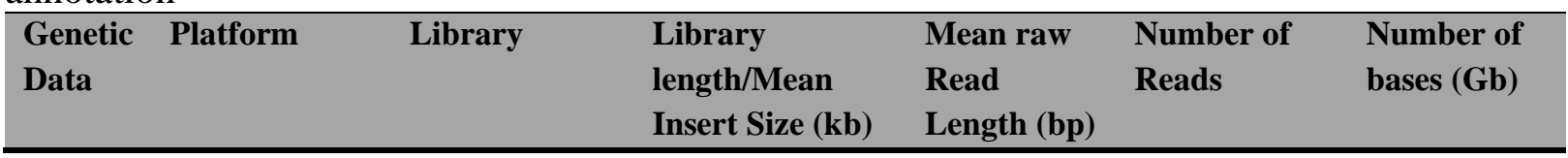




\begin{tabular}{lllllll}
\hline gDNA & Hiseq X Ten & $\begin{array}{l}\text { Paired-end 10x } \\
\text { Chromium }\end{array}$ & $51.7 \mathrm{~kb}$ & 150 & $361,950,449$ & 108.58 \\
gDNA & ONT MinION & Ligation & $47 \mathrm{~kb}$ & 6,417 & $1,225,865$ & 7.865 \\
cDNA & PacBio & Iso-Seq & $\begin{array}{l}\text { Full transcripts } \\
\text { (brain) }(2.6 \mathrm{~kb})\end{array}$ & 12,000 & $20,558,110$ & 38.650 \\
cDNA & PacBio & Iso-Seq & $\begin{array}{l}\text { Full transcripts } \\
\text { (heart + testes) } \\
(2.0 \mathrm{~kb})\end{array}$ & 10,000 & $18,985,944$ & 29.496 \\
\hline
\end{tabular}

401

\section{Improvements to genome assembly completeness during scaffolding}

Sequential steps of scaffolding, polishing, and quality control (Fig. 1, Supplementary

Materials: Fig. S2, Table S3) improved the genome assembly statistics considerably from the initial SUPERNOVA S. vulgaris assembly (Supplementary Materials: Fig. S4). BUSCO

completeness was approximately $94.6 \%$, which was largely achieved by the initial assembly (92.9\%), but somewhat improved over the additional assembly steps (Fig. 2a). The final BUSCO completeness score is comparable to other chromosome-level passerine assemblies on NCBI (Fig. 3a). BUSCO predictions are susceptible to base calling errors and can also fluctuate due to changes elsewhere in the genome assembly (Edwards 2019) (see section 2.8 BUSCO versus BUSCOMP performance benchmarking). As a consequence, BUSCO can under-report the true number of complete BUSCO genes in an assembly (Edwards et al., 2018; Field et al., 2020; Edwards et al., 2021). We therefore used BUSCOMP to compile complete BUSCO genes from across all stages of the assembly. Only 70 (1.4\%) of the 4,915 Aves BUSCO genes were found to be "Missing" from all assembly versions, with 4,764 (96.9\%) rated "Complete" in at least one stage (Fig 3a, BUSCOMP).

The final assembly had the fewest unmapped Iso-Seq reads (Fig. 2b), with the largest improvement seen post gap-filling, followed by chromosome scaffolding. An increase in missing Iso-Seq transcripts was observed after scaffolding with the Iso-Seq reads themselves, and post long-read scaffolding, due to reads no longer partially matching at scaffold ends. 
421 Polishing caused a minimal improvement on the total number of mapped Iso-Seq reads, and

422

423

424

425

426

427

428

none were lost during scaffold clean-up with DIPLOIDOCUS (runmode purgehaplotig and vecscreen). Assessment using GEMOMA and SAAGA revealed that across these assembly steps we see a generally consistent increase in the quality of the predicted proteins during annotation (Fig. 2c), with the largest increases occurring post long-read scaffolding, followed by chromosome scaffolding, and then scaffold clean-up.

Of the 33,454 high-quality isoform transcripts in the PacBio Iso-Seq data, only 241

failed to map to the final genome assembly, a $17.2 \%$ decrease compared to the 291 that failed to map to $S$. vulgaris vNA (Fig. 3b).

\section{Final genome assembly size, heterozygosity, and contiguity}

The $S$. vulgaris vAU assembly of $1,049,838,585$ bp covers approximately $93.78 \%$ of the total estimated $1.119 \mathrm{~Gb}$ genome size (Supplementary Materials: Appendix 2 Validation of SUPERNOVA genome size prediction using JELLYFISH). A similar estimation of genome completeness was reported by K-MER ANALYSIS TOOLKIT (KAT), with the raw read1s (forward reads) estimating a genome completeness of $96.7 \%$ (estimated genome size $1.125 \mathrm{~Gb}$, estimated heterozygosity rate $0.57 \%$ ) and read2s (reverse reads) estimating a genome completeness of $95.92 \%$ (estimated genome size $1.135 \mathrm{~Gb}$, estimated heterozygosity rate 0.54\%) (Supplementary Materials: Fig. S5). Predicted genome sizes based on either read1s or read2s using KAT were slightly larger than the estimation generated by JELLYFISH using all the read data, however the length range was relatively consistent (1.119-1.135 Gb). This assembly reports a scaffold N50 of $72.5 \mathrm{Mb}$ and L50 of 5, with a total of 1,628 scaffolds (Table 2); $98.6 \%(1,035,260,756 \mathrm{bp})$ of the sequence length has been assigned to the 32 putative nuclear chromosomes (identified via the $T$. guttata v3.2.4 assembly), plus a mitochondrial genome. The final assembly contains 14 macrochromosomes (> $20 \mathrm{Mb}$, as 
445 described in Backström et al., 2010), with relative sizes appearing in consensus with known

446 karyotype of S. vulgaris (Calafati \& Capanna 1981). Macrochromosome scaffolds account for

$44781.9 \%$ of the total assembly size, with the remainder on microchromosomes $(16.9 \%)$ or

448 unplaced scaffolds. While these large scaffolds remain only putative chromosomes assuming

449 karyotype orthology until they can be validated with further read data, increased completeness

450 scores post chromosomal alignment across all assembly assessment metrics (Fig. 2) support

451 the assembly structure.

a)

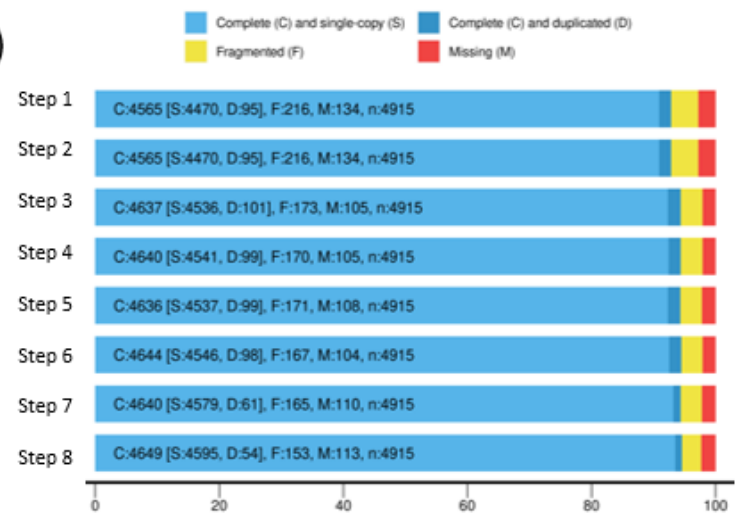

b)

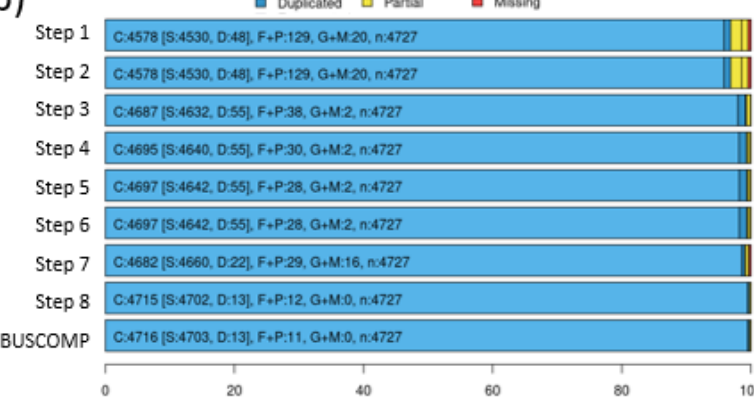

c)

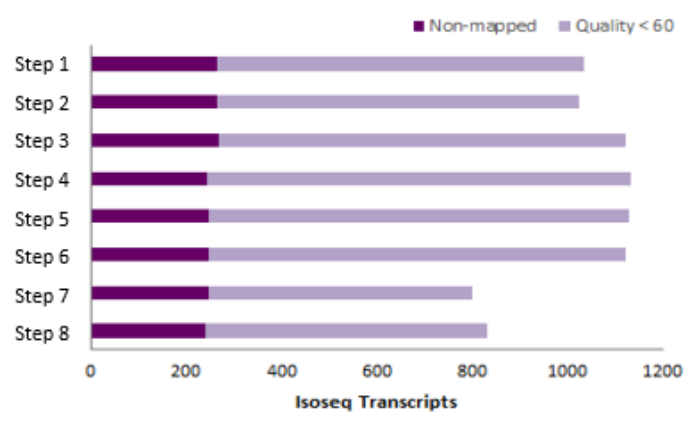

d)

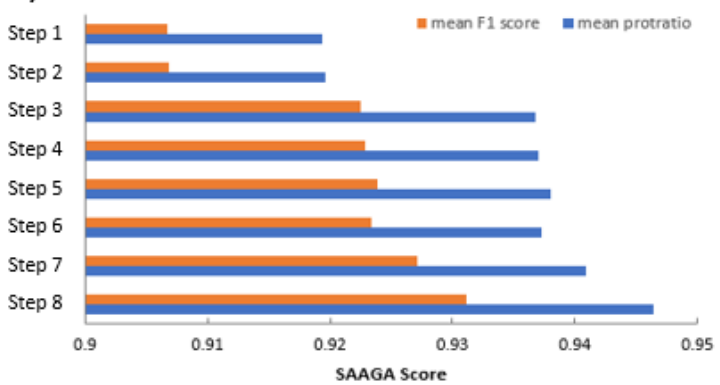

Figure 2: Sturnus vulgaris vAU assembly steps overview. Quality and completeness assessments for eight sequential assembly steps: step 1 (SUPERNOVA assembly), step 2 (DIPLODOCUS primary assembly), step 3 (SSPACE-LONGREADS scaffolding), step 4 (GAPFINISHER gapfilling), step 5 (L_RNA_SCAFFOLDER), step 6 (PILON polishing), step 7 (DIPLODOCUS clean up), and step 8 (SATSUMA2 Chromosome scaffolding). a) BUSCO (Aves, $\mathrm{n}=4,915$ ) completeness rating summaries for the sequential steps of $S$. vulgaris genome assembly. b) BUSCOMP completeness results for the 4,727 BUSCO genes identified as single copy and complete in one or more assembly stages. The final BUSCOMP row compiles the best rating for each gene across all eight steps. c) The number of Iso-Seq reads that failed to map to each assembly step. d) SAAGA annotation scores of mean protein length ratio (blue) and F1 score (orange) (see Methods for details). 
bioRxiv preprint doi: https://doi.org/10.1101/2021.04 07.438753 t this version posted May 26 2021. The copyright holder for this preprint (which was not certified by peer review) is the author/funder, who has granted bioRxiv a license to display the preprint in perpetuity. It is made available under aCC-BY-NC-ND 4.0 International license.

465

466

Table 2: Sturnus vulgaris overview of assembly statistics for vAU1.0, vAU1.1, and vNA, assessed using BUSCOMP.

\section{Sturnus vulgaris Sturnus vulgaris Sturnus vulgaris}

vAU1.0

vAU1.1

\begin{tabular}{llll}
\hline Total length (bp) & $1,049,838,585$ & $1,043,825,671$ & $1,036,755,994$ \\
Number of scaffolds & 1,628 & 1,344 & 2,361 \\
Scaffold N50 (bp) & $72,525,610$ & $72,244,370$ & $3,416,708$ \\
Scaffold L50 & 5 & 5 & 89 \\
Largest scaffold (bp) & $151,927,750$ & $151,503,485$ & $11,828,398$ \\
Mean scaffold length (bp) & $644,864.0$ & $776,656.01$ & $439,117.3$ \\
$\begin{array}{l}\text { Median scaffold length } \\
\text { (bp) }\end{array}$ & 1,337 & 1,343 & 4,856 \\
Number of Contigs & 23,815 & & \\
Contig N50 (bp) & 145,864 & 23,340 & 22,666 \\
Contig L50 & 2,030 & 147,322 & 147,183 \\
Gap (N) length (bp) & $13,242,113(1.26 \%)$ & $0.74 \%$ & 1,908 \\
$\begin{array}{l}\text { GC (Guanine-Cytosine) } \\
\text { content (\%) }\end{array}$ & $41.73 \%$ & $41.72 \%$ & $23,939,528(2.31 \%)$ \\
\hline
\end{tabular}

a)

BUSCO Assessment Results

Complete (C) and single-copy (S)

Missing (M)

b)

S. vulgaris vAU1.0

S. vulgaris VAU1.1

S. vulgaris VNA

Taeniopygia guttata

Passer domesticus

Calypte anna

Parus major
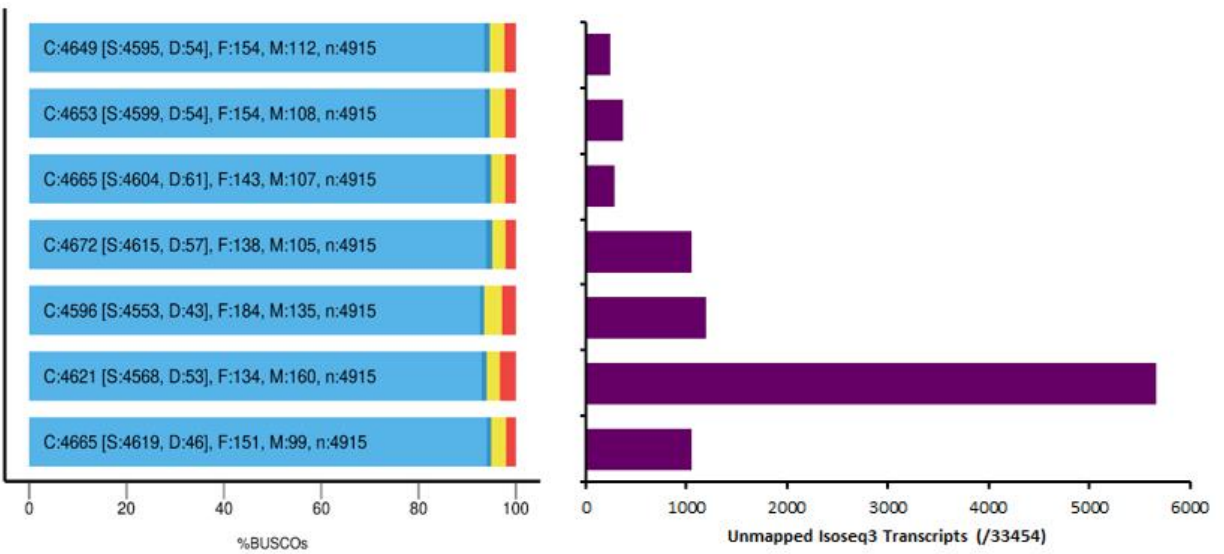

467

468

469

470

471

472

473

474

475

Figure 3: Assessment of Sturnus vulgaris and comparison avian reference assemblies. a) BUSCO (Aves) assessments of assembly completeness of $S$. vulgaris vAU1.0, and the NCBI uploaded genome $S$. vulgaris vAU1.1, presented alongside $S$. vulgaris vNA and four recent high-quality avian reference genomes (Taeniopygia guttata assembly accession GCF_008822105.2, Passer domesticus assembly accession GCA_001700915.1, Calypte anna assembly accession GCA_003957555.2, Parus major assembly accession GCA_001522545.3). b) Total number of Iso-Seq transcripts that failed to map to each assembly. 


\subsection{Sturnus vulgaris vAU whole transcriptome data analysis}

478 subreads) (Table 1). This produced a total of 33,454 clustered high-quality (predicted

479 accuracy $\geq 0.99$ ) reads, and 157 clustered low-quality (predicted accuracy < 0.99 ) reads

480 (Supplementary Materials: Table S4). These high-quality read data were used to improve the 481 scaffold assembly of the genome using L_RNA_SCAFFOLDER (see section 2.1) and assess 482 genome completeness (using count comparison of unmapped Iso-Seq reads, see section 483 2.5.2). After being passed through the TAMA collapse pipeline, a total of 28,448 non484 redundant transcripts were retained to create the final S. vulgaris vAU transcriptome, which 485 was used for gene prediction when completing the annotation of the genome assembly. This 486 final three tissue (brain, gonad, heart) Iso-Seq transcriptome had a moderate level overall 487 BUSCO completeness of around 63\% that compares to other avian Iso-Seq transcriptomes 488 (Fig. 4a), with a wide range of gene ontology terms identified in the final Iso-Seq transcript 489 list (Fig. 4b) that resembled other avian Iso-Seq GO term distributions (Yin et al., 2019). 


\section{a)}

S. vulgaris (short read 1 tissue)

S. vulgaris $H Q$ reads (Iso-Seq 3 tissue: 33454 reads)

S. vulgaris Tama collapse (Iso-Seq 3 tissues: 28448 reads)

S. vulgaris combined (Iso-Seq HQ \& short read liver)

Anas platyrhynchos (Iso-Seq 8 tissues)

Calypte anna (Iso-Seq 1 tissue)

\section{b)}

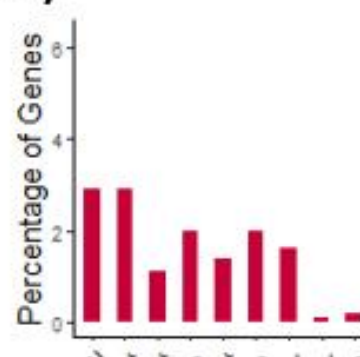

BUSCO Assessment Results
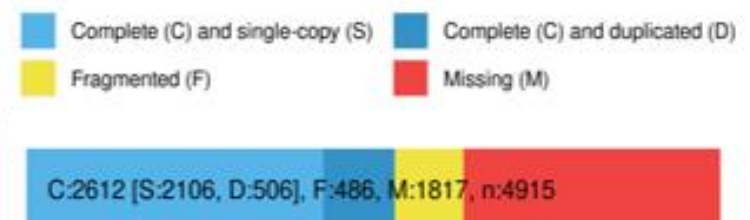

C.3245 [S:1100, D:2145], F:109, M:1561, n:4915

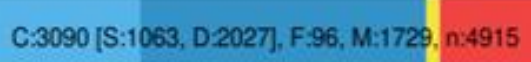

C:3664 [S:962, D-2702], F:236, M:1015, n:4915

C:4064 [S:1145, D:2919], F-251, M:600, n:4915

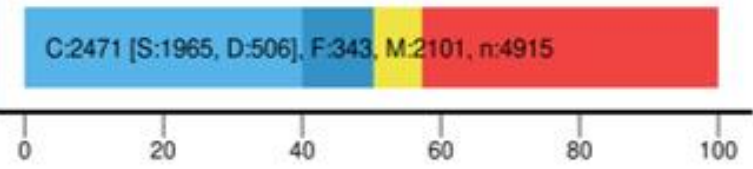

\%Buscos
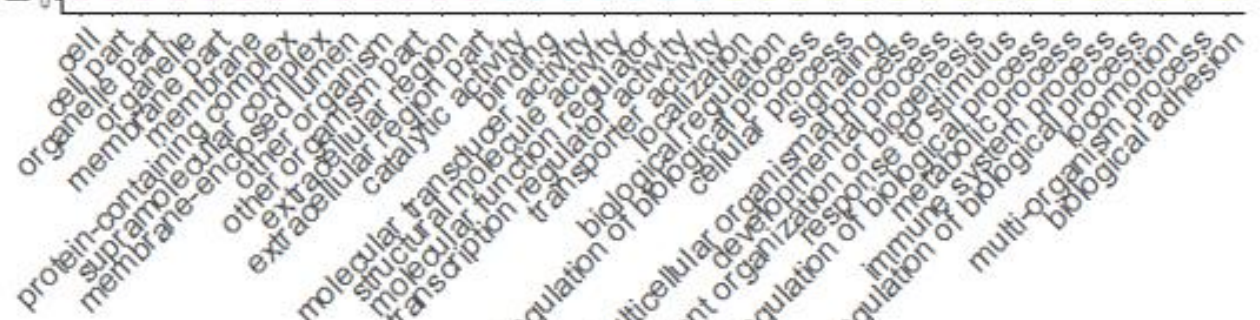
transcriptome. a) BUSCO (aves) rating summaries for $S$. vulgaris short read liver transcriptome, the high-quality Iso-Seq $S$. vulgaris transcript produced though the Iso-Seq v3.3 pipeline, the final $S$. vulgaris transcriptome produced by TAMA collapse pipeline, and combined high-quality Iso-Seq and short read liver transcripts, alongside two other avian IsoSeq transcriptomes (Anas platyrhynchos using pectoralis, heart, uterus, ovary, testis, hypothalamus, pituitary and 13 days-old embryo tissue (Yin et al., 2019), and Calypte anna using liver tissue (Workman et al., 2018)). b) Breakdown of major GO terms in the sequenced 
501

502

503

504

505

506

507

508

509

510

511

512

513

514

515

516

517

\subsection{Sturnus vulgaris genome annotation}

The initial annotation produced by GEMoMA, informed by the 26 avian genome annotations available at the time on Ensembl (Supplementary Materials, Table S1), predicted 21,539 protein coding genes, with 97.2\% BUSCO completeness (93.1\% complete when longest protein-per-gene extracted with SAAGA) (Fig. 5). The initial MAKER2 annotation reported 13,495 genes, and a BUSCO completeness of 79.5\% (Fig. 5). The merged final annotation reported a BUSCO completeness of 98.2\% (Fig. 5a), and this annotation predicted a total of 21,863 protein-coding genes and 79,359 mRNAs. There was an average of 10.7 exons and 9.7 introns per mRNA, with an average intron length of 3,364 (Table 3). Of these, 1,764 are single-exon genes and 2,330 single-exon mRNA. Predicted coding sequences make up $5.4 \%$ of the assembly, with $44.77 \%$ remaining outside any gene annotation (Fig. 5b).

The predicted transcripts were mapped using SAAGA to the Swiss-Prot database, with 66,890 transcripts returning successful hits $(84.3 \%)$ and 12,469 transcripts remaining unknown (15.7\%) for the final annotation (Fig. 6a). The known proteins had an average length of 652 amino acids (aa) and the unknown proteins had an average length of 426 aa (Fig. 6a). Most of the predicted proteins were of high quality, with around $56 \%$ of them having an F1 score (see Methods) of greater than 0.95 (Fig. 6b). Similar results were seen when the Gallus gallus reference proteome was used, with 69,714 known proteins of average length of 646 aa, 9,645 known proteins of average length of 401 aa, and the final merged annotation having the same F1 score distribution (Fig 6c \& 6d).

The GEMoMA annotation had similar protein quality patterns, with 57,026 known proteins (average length 664 aa), and 10,400 unknown proteins (average length 401 aa) (Fig. 6e). The MAKER2 displayed much greater similarity in protein length histogram between known and unknown proteins, with shorter proteins with known homologs (average length 
525565 aa), but longer unknown proteins (average length 549 aa) (Fig. 6f). The S. vulgaris vNA

526 annotation final merged annotation had extremely similar statistics to the final S. vulgaris

527 vAU annotation, with an average known protein length of 650 aa, and an average unknown

528 protein length of 407 aa (Supplementary Materials: Figure S2b).

\section{$529 \quad 3.4$ Sturnus vulgaris genome-wide patterns of genomics features}

530 Transcript density compared between mapped Iso-Seq reads and predicted transcripts

531 in the final annotation displayed similar patterns, with some minor variation in patterns

532 between the two tracks (Fig. 7; track 1). Final predicted gene densities (Fig. 7; track 2) were

533 largely following the patterns seen in transcript densities. Further, patterns of transcript and

534 gene numbers across the genome track relatively consistently to GC content (Fig. 7; track 4).

535 Global whole genome variant data (Fig. 7; track 3) revealed genomic regions where

536 variant density is low or non-existent, indicative of high genetic conservation across the

537 species, and genomic regions where variant density peaks are indicative of variant hotspots.

538 Interestingly, we see regions of high conservation corresponding to peaks in gene and/or

539 transcript numbers (e.g., midway through chromosome 4), which may be indicative of regions

540 of highly conserved genes and possibly centromere locations. 
Table 3: Summary of genome annotation of Sturnus vulgaris vAU and vNA assemblies.

\begin{tabular}{llll}
\hline Genome Annotation & S. vulgaris vAU & S. vulgaris vNA \\
\hline Genes & Total number & 21,863 & 21,944 \\
& Average length & $34,699 \mathrm{bp}$ & $35,761 \mathrm{bp}$ \\
& mean mRNAs per gene & 3.6 & 3.7 \\
mRNA & Total number & 79,359 & 81,714 \\
& Average length & $38,073 \mathrm{bp}$ & $37,857 \mathrm{bp}$ \\
& mean exons per mRNA & 11.8 & 11.8 \\
CDS & Total number & 79,359 & 81,714 \\
& Average length & 1,851 & 1,836 \\
& Average intron in CDS & 3,364 & 3,343 \\
& length & & \\
Exons & Total number & 933,014 & 962,220 \\
& Mean length & 163 & 158 \\
Gene Function & Ontology Term & $60.26 \%(13174 / 21863)$ & $59.68 \%(13097 / 21944)$ \\
& InterPro & $78.87 \%(17244 / 21863)$ & $77.57 \%(17022 / 21944)$ \\
& SUPERFAMILY & $60.36 \%(13197 / 21863)$ & $58.26 \%(12786 / 21944)$ \\
\hline
\end{tabular}

555 


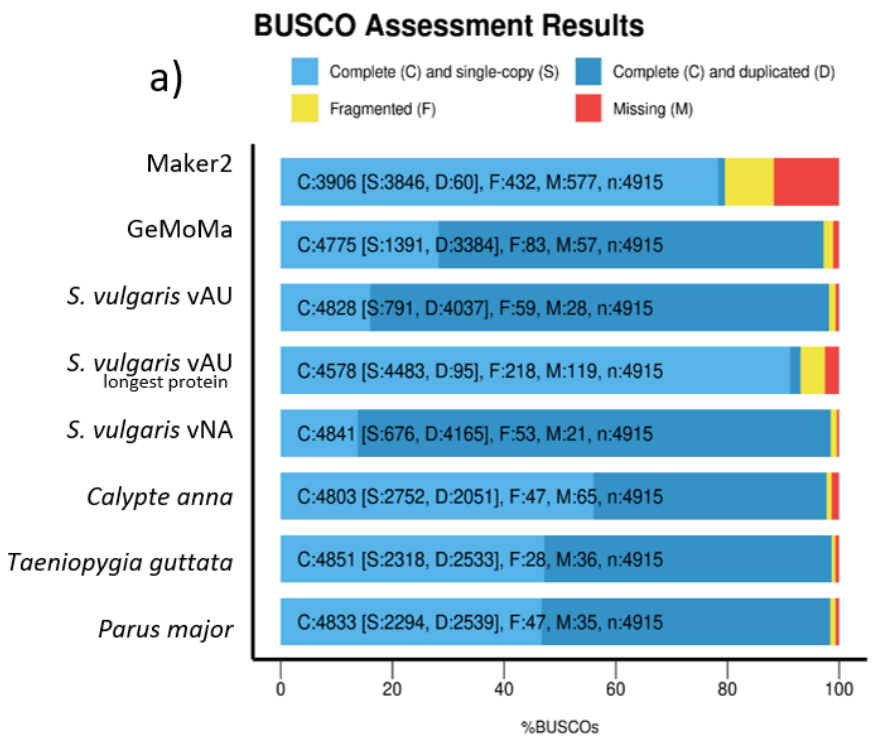

b)

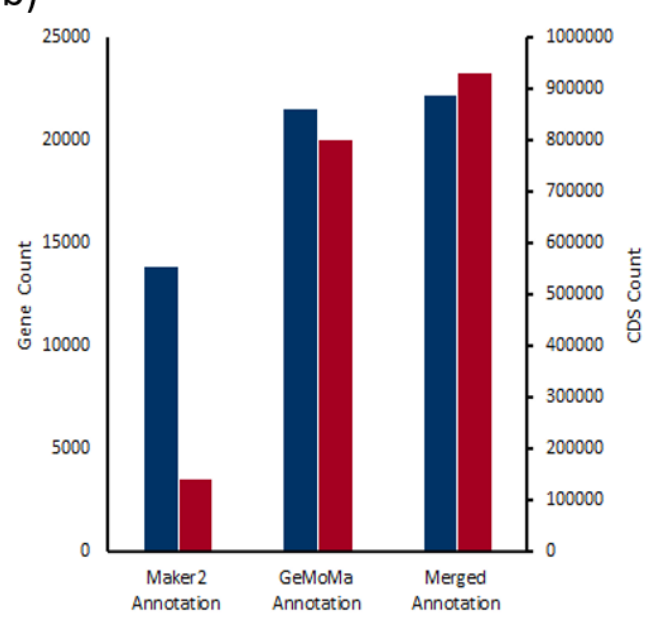

Figure 5: Sturnus vulgaris assessment of annotation. a) BUSCO (Aves) assessments of initial MAKER2 and GEMOMA assemblies, the final $S$. vulgaris vAU annotation, the final annotation with the longest protein-per-gene extracted using SAAGA, the final S. vulgaris vNA annotation (combined GEMOMA and MAKER2 annotation), and the ensemble annotations of three additional passerines. b) The number of genes (blue) and CDS (red) in the MAKER2 annotation, GEMOMA annotation, and merged annotation. 


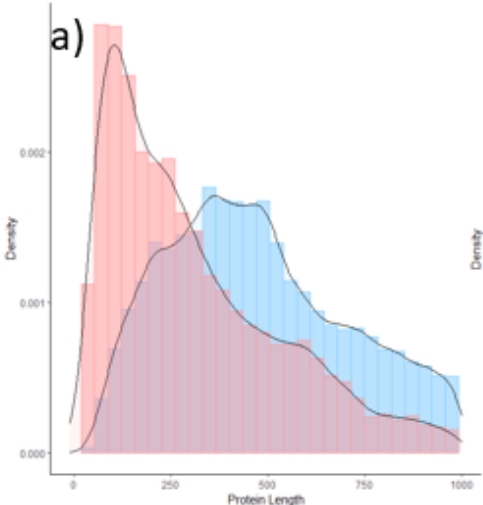

b)
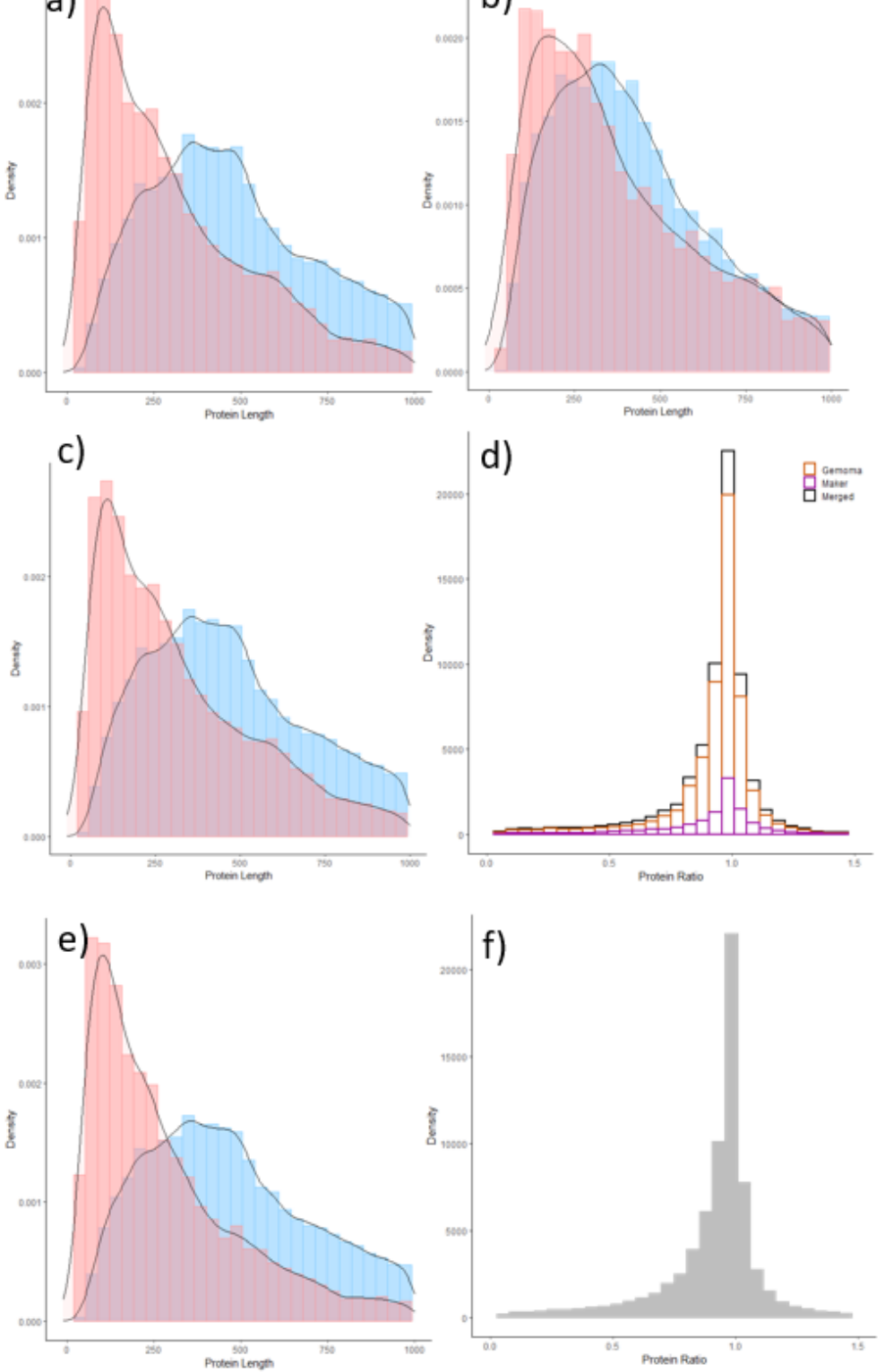

Figure 6: Summary of predicted annotated proteins. a) Protein lengths for known proteins (blue, with a located Swiss-Prot comparison) and unknown proteins (red, those that did not map to Swiss-Prot) for the GEMoMA annotation compared to Swiss-Prot. b) Protein lengths of known and unknown proteins for the MAKER2 annotation compared to Swiss-Prot. c) Protein lengths of known and unknown proteins for the merged GEMOMA and MAKER2 annotation compared to Swiss-Prot. d) Protein length ratio between output from SAAGA for all known Swiss-Prot proteins (where a score close to 1 indicates a high-quality gene annotation, protein length ratio calculated as annotated protein length / best Swiss-Prot reference protein length) (merged annotation $=$ black, GEMoMA annotation $=$ orange, MARKER2 annotation = purple). e) Protein lengths of known and unknown proteins for the merged GEMOMA and MAKER2 annotation compared to Gallus gallus reference proteome (UP000000539_9031). f) Protein length ratio between output from SAAGA for the merged annotation against the Gallus gallus reference proteome. 
bioRxiv preprint doi: https://doi. org/10.1101/2021.04.07.438753. this version posted May 26, 2021. The copyright holder for this preprin (which was not certified by peer review) is the author/funder, who has granted bioRxiv a license to display the preprint in perpetuity. It is made available under aCC-BY-NC-ND 4.0 International license.

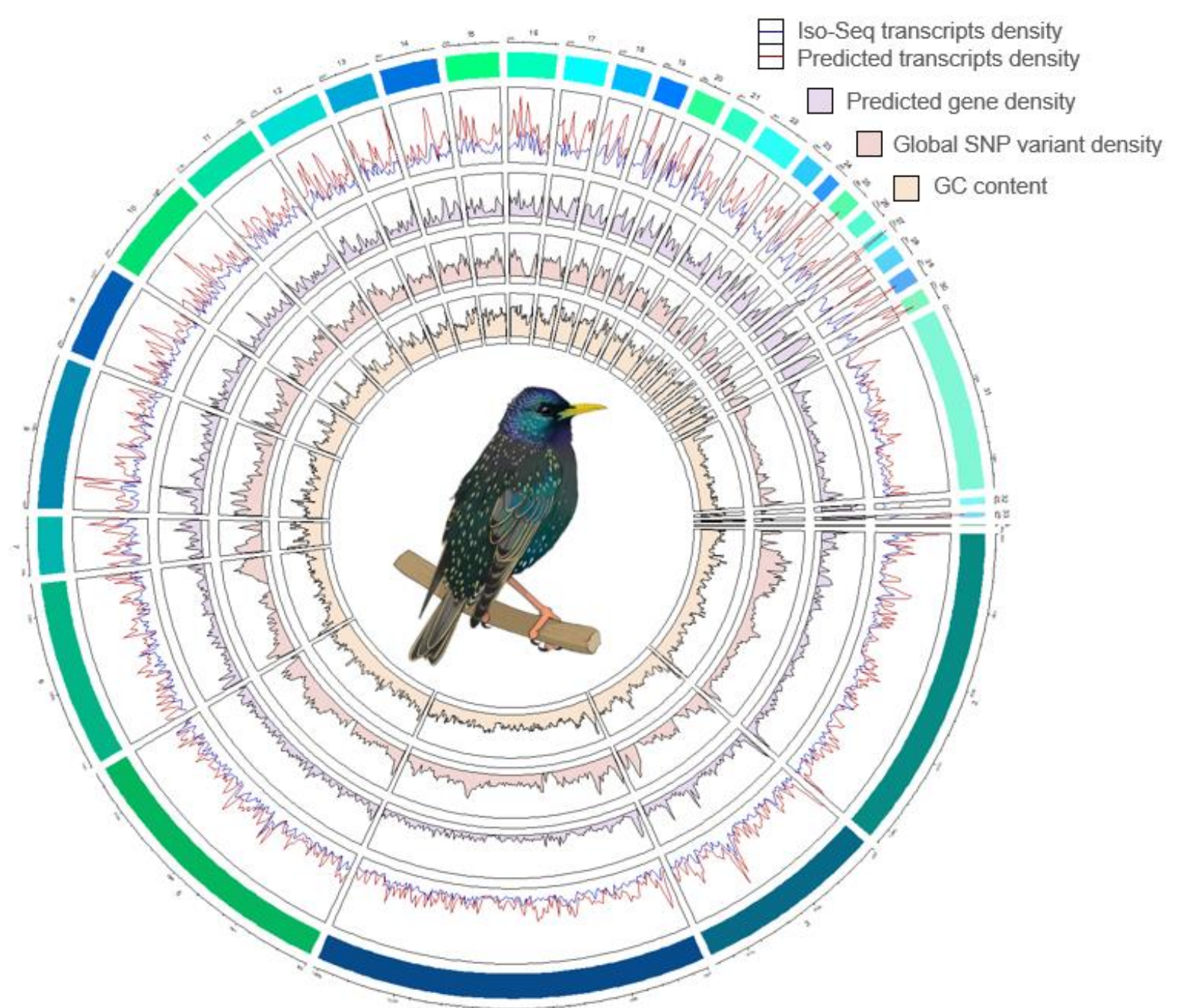

Figure 7: CIRCLIZE plot of the 33 main chromosomal scaffolds (32 putative autosomes plus mtDNA) in the Sturnus vulgaris (S. vulgaris vAU) genome assembly ( $>98 \%$ of the total assembly length). The tracks denote variable values in 1,000,000 bp sliding windows. From the outermost track in, the variables displayed are track 1 (Iso-Seq transcripts as blue line, final annotation transcripts as red line), track 2 (final annotation gene counts, purple area), track 3 (variant density, red area), and track 4 GC content (yellow area). 
602

603

604

605

606

607

608

609

610

611

612

613

614

615

616

617

618

619

620

621

622

623

624

625

\subsection{BUSCO versus BUSCOMP performance benchmarking}

For the non-redundant pseudodiploid S. vulgaris vAU SUPERNOVA assembly, BUSCOMP revealed differences in the BUSCO ratings of scaffolds dependent on the assembly background (Fig. 8). Despite the primary ('pri') assembly being a subset of the nonredundant pseudodiploid ('dinpnr') assembly, it identified more "Complete” BUSCO genes (4,565 versus 4,532) with fewer "Missing" (131 versus 171) (Fig. 8a). The alternative assembly ('alt') subset similarly returned a partially overlapping set of BUSCO genes with 'dipnr', including some not found in 'dipnr' or 'pri': in total, only 101 genes were missing from all three assemblies. Reducing the primary assembly to the 968 (of 18,439) scaffolds containing a complete BUSCO gene ('pribusco'), increased the number of complete genes from 4,565 to 4,586 and reduced the number missing from 131 to 112 . Most unexpectedly, reverse complementing these scaffolds reduced the number of BUSCO genes rated

"Complete" by two, and increased the number "Missing" by fifteen (Fig. 8a). All five assemblies returned complete BUSCO genes that were fragmented or missing in all the other four assemblies (Supplementary File 1, BUSCOMP v3 results), for a combined total of 4,760 complete and only 74 missing.

Adding direct or reverse-complemented copies of the 'pribusco' scaffolds increased the number of "Duplicated" genes, but still returned single copy complete genes (Fig. 8b). Doubling and then tripling the assembly size also increased the number of "Missing" genes from 112 to 198 ('duplicate') and then 207 ('triplicate'). As before, these summary numbers hide some gene gains as well as gene losses; only 77 genes are missing from all three BUSCO runs, with 4,750 returned as complete by at least one. Adding randomly shuffled versions of the 'pribusco' scaffolds only had a marginal effect on BUSCO ratings, with four ('2n') to five (‘3n’, ‘ $4 n$ ’) fewer complete genes returned and seven additional genes missing following 
addition of the random sequences (Fig. 8c). Ten replicate analyses of the `pribusco`scaffolds returned identical results (Supplementary File 1, BUSCOMP v3 results).

629 with the primary assembly returning the same numbers of complete, partial/fragmented and

630 missing genes as the pseudodiploid assembly (Supplementary File 1, BUSCOMP v3 results).

631 Similarly, reverse complementing scaffolds or increasing genome size gives no difference to

632 the completion statistics. Unlike BUSCO, BUSCOMP rates 100\% of complete BUSCO genes

633 for duplicated or triplicated scaffolds as 'Duplicate' rather than 'Single Copy'. Most

634 reassuringly, every complete BUSCO gene returned by a variant or subset of the

635 pseudodiploid assembly is also returned as 'Complete' in the pseudodiploid assembly itself.

636 Results using BUSCO v5 and the updated lineage data were qualitatively the same as v3,

637 showing largely identical trends (Supplementary File 2, BUSCOMP v5 results). The

638 exception is that reverse-complementing scaffolds reduced the complete BUSCO genes by

639 one (7,555 to 7,554) and increased the number missing by one (391 to 392). Curiously, this

640 was not reflected by analysis of the duplicated scaffolds, in which all 7,555 'pribusco'

641 complete genes were returned as complete and duplicated. It should be noted that the

642 'pribusco' scaffolds for the v5 analysis are missing a greater proportion of the BUSCOMP-

643 compiled single copy complete BUSCO genes because they were still defined from v3 data.

644 
bioRxiv preprint doi: https://doi.org/10.1101/2021.04.07.438753; this version posted May 26, 2021. The copyright holder for this preprint (which was not certified by peer review) is the author/funder, who has granted bioRxiv a license to display the preprint in perpetuity. It is made available under aCC-BY-NC-ND 4.0 International license.

a) starling10x.dipnr
starling10x.pri
starling10x.alt
Pseudodip
starling10x.pribusco
starling10x.revcomp

Core

$$
\text { b) }
$$

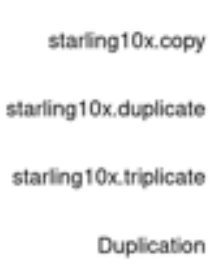

Duplication

c)

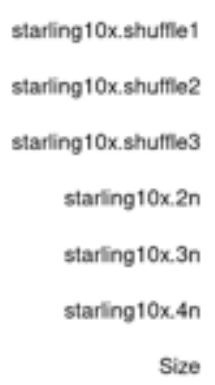

\section{C:0 [S:0, D:0), F.0, M.:4915, n:4915}

C:0 [S:0, D:0], F:0, M:4915, n:4915

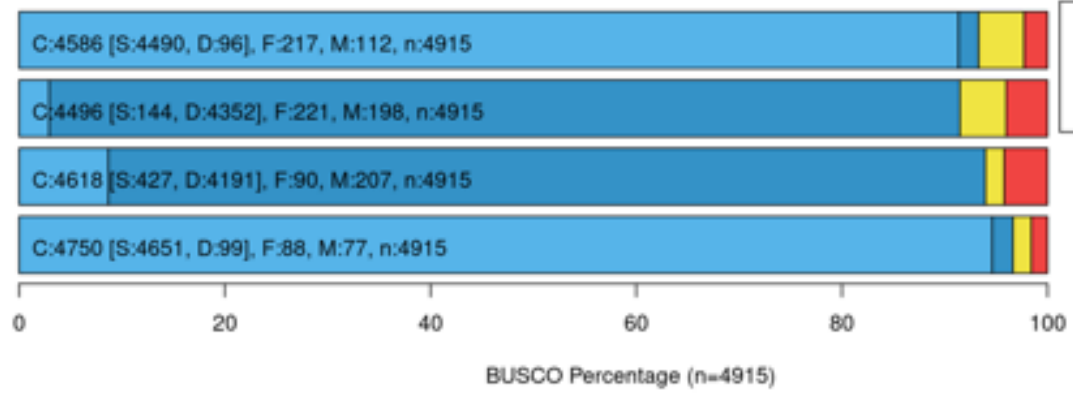

\section{Size BUSCO Rating Summary}

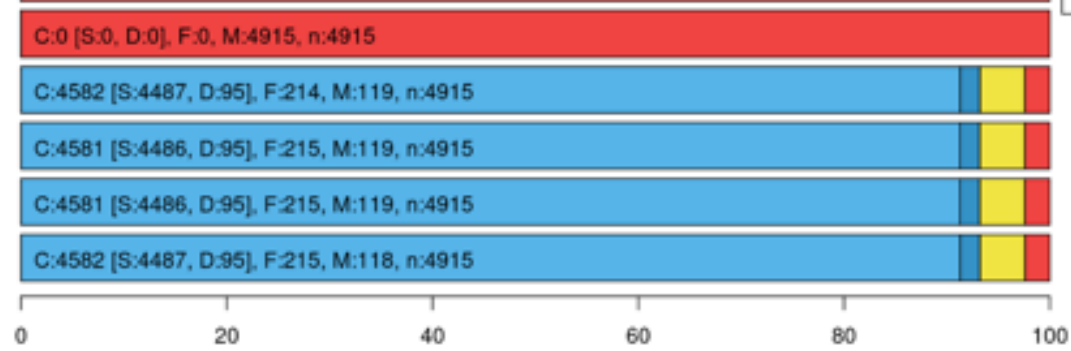

Duplication BUSCO Rating Summary

Pseudodip BUSco Rating Summary

\section{Complete \\ Duplicated \\ ㅁ Fragmented}

- Missing

\section{Busco Percentage (n=4915) \\ Figure 8. Compiled BUSCO results for benchmarking data.}




\section{Discussion} genome assembly length to 32 putative nuclear chromosome scaffolds. We demonstrate the utility of both transcripts and gene annotation in validating S. vulgaris vAU assembly processes. BUSCOMP, Iso-Seq transcript, and SAAGA annotation assessment were largely in complementary assessment approaches in ensuring that aspects of genome quality are not sacrificed to improve non-specific assembly quality metrics, such as N50. We also present a second, North American, genome assembly, S. vulgaris vNA (GCF_001447265.1). Overall, the $S$. vulgaris vAU assembly improved genome assembly statistics over the $S$. vulgaris vNA genome, with a greater percentage of the estimated $1.119 \mathrm{~Gb}$ genome represented $(94 \% \mathrm{vs}$ 93\%), an increase of scaffold $\mathrm{N} 50$ from 3.42 Mb to $72.5 \mathrm{Mb}$, and a decrease in scaffold L50

668 from 89 to 5. The S. vulgaris vNA still has good assembly statistics (Table 2, Table S3) and 669 has a marginally higher BUSCO completeness (Fig. 3a) and BUSCOMP completeness

670 (Supplementary Materials: Fig. S6) of approximately 20 BUSCO sequences. There is

671 increasing recognition of the importance of pan-genomes (genome assemblies that

672 differentiate between genes/regions shared by all members of the species, and dispensable or 673 rare genes/regions) (Hirsch et al., 2014; Sherman \& Salzberg 2020), which are essential for 674 many model organisms (Vernikos et al., 2015). Having these two high-quality de novo 675 assemblies from different populations will improve future genomic work on the global 676 invasive populations of this species, and facilitate review of structural variation (e.g., 677 inversions) that may exist across different populations. It should be noted, however, that the 
678 final scaffolding step for $S$. vulgaris vAU assumed structural conservation between the

679 starling and zebra finch and thus future synteny analyses may want to use the earlier assembly

680 step.

$681 \quad 4.1$ BUSCO and BUSCOMP assembly completeness assessment

BUSCO (Simão et al., 2015) is an extremely useful and widely-used used assembly

683 assessment tool, providing information on which conserved lineage specific genes are present,

684 fragmented, or absent from a genome assembly. The program, however, can suffer from

685 inconsistent BUSCO gene identification, where a particularly BUSCO may be dropped from a 686 report due to changes elsewhere in the assembly (Edwards 2019), which can result in under-

687 reporting of assembly completeness (Edwards et al., 2018; Field et al., 2020; Edwards et al.,

688 2021). Here, we confirm this behaviour on benchmarking datasets derived from the $S$.

689 vulgaris vAU pseudodiploid 10x linked read assembly (Supplementary Materials: Fig. S3, 8).

690 Adding and removing scaffolds can both alter the BUSCO ratings for "Complete" genes

691 within the unchanged scaffolds (Fig. 8, Supplementary File 1, BUSCOMP v3 results,

692 Supplementary File 2, BUSCOMP v5 results). Many of these changes are likely to be the

693 consequence of changes in score thresholds and/or gene prediction models. However, we also

694 demonstrate some unexpected behaviours that are harder to explain, such as changes to

695 BUSCO gene ratings when scaffolds are reverse complemented (Fig 8a).

696 This unpredictable variability in the identification of BUSCOs across genome

697 assembly versions poses some obvious challenges when trying to compare alternate versions

698 of the same assembly. This is particularly true when trying to interpret small changes in

699 BUSCO ratings as assemblies near completion. In addition, an important feature of BUSCO is

700 that it incorporates sequence quality in the context of the gene prediction models it generates.

701 This is desirable for assessing final assembly quality, but can present problems when 
comparing early assembly stages, prior to error-correction by “polishing”. BUSCOMP

(https://github.com/slimsuite/buscomp) is robust to differences in assembly size, base-calling quality, and rates the "completeness potential" of an assembly based on the presence of genes first identified for that species by BUSCO. Here, we used BUSCOMP analysis of sequential assembly steps to gain a more accurate understanding of how assembly decisions affected genome completeness (Fig. 2, Supplementary Materials: Fig. S4). BUSCOMP analysis can then be complemented by other tools, such as KAT (Mapleson et al., 2017), SAAGA (https://github.com/slimsuite/saaga), and BUSCO itself to get additional assessment of sequence quality.

\subsection{Transcript- and annotation-guided Sturnus vulgaris vAU genome assembly}

The assembly of the $S$. vulgaris vAU genome was improved by assessing mapped IsoSeq whole transcripts and quality scores of predicted proteins from homology-based annotation. Mapping of the high-quality Iso-Seq reads proved to be an extremely fast method of assessment (33,454 Iso-seq sequences mapped in $<5$ mins with 16 CPU cores), while the GEMOMA and SAAGA compute time of $12 \mathrm{hrs}$ per assembly was roughly comparable to BUSCO (approximately $50 \mathrm{CPU}$ hrs per assembly on an average machine), though more computationally intensive (GEMoMA ran for approximately 200 CPU hours per assembly, and SAAGA ran for approximately 8 CPU hours per assembly). Over the eight sequential assembly steps, there was a decrease in unmapped Iso-Seq reads, indicating improved sequence representation, with gap-filling yielding the greatest change. Similarly, the quality of annotated proteins predicted by GEMOMA, as assessed by SAAGA, demonstrated ongoing improvements through ONT scaffolding, clean-up, and chromosome alignment. It is also noteworthy that increases in large-scale sequence connectivity using the T. guttata genome (Peona et al., 2018) improved the assembly's performance across all metrics, including 
completeness estimates, although future Hi-C analysis will be required to confirm the predicted genome structure.

Further, BUSCOMP provided an important means of standardising BUSCO

annotation ratings across the multiple assembly steps. This method, together with the mapped Iso-Seq reads, can deal with the unpolished intermediary genome steps, and does not suffer the same sequence identification accuracy issues as the traditional stand-alone BUSCO analysis. Together, the standardised assessment reported by BUSCOMP, and the comprehensive and genome/species specific set of genes provided by Iso-seq and GEMoMA/SAAGA showcase the complementary features of these annotation approaches for assembly assessment.

\subsection{Improvements to contiguity and completeness during Sturnus vulgaris vAU genome} assembly

Several alternative assembly pipelines were assessed (Supplementary Materials: Fig. S2), with upstream assembly decisions based primarily on establishing reasonable base assembly statistics (scaffold N50, scaffold L50, contig numbers). Assembly size increased during scaffolding steps, due to estimated bases in gaps, while a decrease in assembly size was only seen during scaffold clean up using Purgehaplotigs. Of all the scaffolding steps, scaffolding with the low coverage ONT long reads resulted in the greatest decrease of scaffold L50 (146 scaffold to 39 scaffold, Supplementary Materials: Fig. S4d) and total scaffold number (18,439 scaffolds to 7,856 scaffolds, Supplementary Materials: Fig. S4a). It has previously been shown that even low coverage of ONT data in conjunction with 10x may produce high-quality genome assemblies (Ma et al., 2019). This was true for our data, which demonstrates the utility of even low coverage, long read sequencing (approximately $4.5 \%$ coverage based on the estimated genome size of $1.119 \mathrm{~Gb}$ ) in greatly improving the 
contiguity of scaffolds generated by short read genome assemblers (though Hi-C data may

(Supplementary Materials: Fig. S4), the Iso-Seq reads were nevertheless were able to scaffold

This long-read transcript scaffolding served to minimise the number of fragmented genes in

the final assembly, helping downstream analysis and gene prediction models. The final assembly maintained reasonably short contig N50 and high contig L50, which will only be improved with much more extensive long-read sequencing of the species. Nevertheless, scaffolding the $S$. vulgaris genome against that of $T$. guttata was able to further scaffold the genome to a predicted chromosome level, assigning $98.6 \%$ of the assembly to previously characterised chromosomes. In support of the assumed synteny of this step, we saw small increases in assembly quality and completeness metrics. 
sequence duplication when comparing to raw read k-mer counts (Supplementary Materials:

775

776

777

778

779

780

781

782

783

784

785

786

787

788

789

790

791

792

793

794

795

796

797 Fig. S5).

\subsection{Sturnus vulgaris vAU transcriptome}

When comparing the completeness of this new starling transcriptome data to existing Illumina short read transcript data produced using liver tissue (Richardson et al., 2017), we see an increase of about $20 \%$ in BUSCO completeness, with a particularly large increase in the number of duplicated BUSCO, a result of the alternate transcript isoforms captured through the Iso-Seq. Assessing the effect the TAMA pipeline had on BUSCO completeness, we see a small drop in complete BUSCOs (Fig. 2a) that appear to have been lost during the mapping to genome assembly step. Finally, comparing our final transcriptome to two other avian Iso-Seq transcriptomes gives an indication of how much unique transcript information is added by the addition of tissues into pooled Iso-Seq sequencing runs. The single tissue IsoSeq liver transcriptome of Calypte anna (Anna's hummingbird) (Workman et al., 2018) reported similar BUSCO completeness to the short read S. vulgaris liver transcriptome. The eight tissue Iso-Seq transcriptome of Anas platyrhynchos (mallard) (Yin et al., 2019) yielded an increase of $30 \%$ in complete BUSCOs, consistent with the expectation that our three-tissue Iso-Seq library will be missing a number of tissue-specific genes.

\subsection{Sturnus vulgaris genome annotation}

Of the approximately 22,000 genes reported in the final annotation, $65 \%$ were from GEMOMA, and 35\% from MAKER2, with the source being randomly selected for common annotation. MAKER2 predicted a higher number of genes in $S$. vulgaris vNA versus $S$. vulgaris vAU $(15,150$ vs 13,495$)$, while GEMOMA predicted a higher number of genes in the $S$. vulgaris vAU genome $(21,539$ vs 20,414). The ratio in predicted MAKER2 and GEMOMA was more biased towards the homology-based predictor, with an approximate ratio of 1:5 between 
MAKER2 and GEMOMA (Fig. 5b). Merging of the MAKER2 annotation to the GEMOMA annotation resulted in an increase in 1.1.\% in BUSCO completeness. Duplication levels were much higher in the GEMOMA annotation when compared to MAKER2 (Fig. 5a). This is not unreasonable, as the GEMOMA annotation will be biased toward well-characterised genes and so may contain more transcripts per gene (Fig. 5b), whereas MAKER2 will inform the prediction of more taxon or possibly species-specific coding sequences. High congruence between Iso-Seq and predicted transcript numbers indicate regions of accurate annotation predictions (Fig. 7). In contrast, Iso-Seq transcripts that are dissimilar or much lower to the predicted transcript densities, are either genomics regions producing tissue specific transcripts

807 not captured by their brain, testes, or muscle, or more likely annotated transcript 808 overprediction.

For the final $S$. vulgaris vAU annotation, the predicted proteins of unknown origin

810 (those that failed to map to Swiss-Prot database or Gallus gallus proteome) had a smaller 811 average length than those with known homologs (Fig. 6a \& 6c). Similar results were found 812 when this approach was used to assess genes predicted in the $R$. marina genome assembly

813 (Edwards et al., 2018), and are indicative that these 'unknown' proteins are fragmented and 814 lower quality predictions that may be due to underlying assembly issues with contiguity or 815 frameshifting indels. The poorer quality could also reflect low stringency MAKER2 gene 816 predictions or homology based GEMOMA annotation of low-quality reference genes. The 817 known proteins predicted by MAKER2 (Fig. 6f) were of apparent lower quality than those 818 reported by GEMOMA as indicated by their shorter lengths and lower protein ratios (Fig. 6e), 819 which may be a result from a combination of incorrect gene predictions, and the high-quality 820 reference homologs inflating quality scores of the GEMOMA annotation in comparison. 
824 increased quality of unknown protein predictions in the vAU annotation, possibly due to the more Iso-Seq data mapping to the vAU genome (Fig. 4b) or the higher contiguity. Predicted genes were more commonly shorter than their closest reference protein hits, indicative there might still be some truncated gene predictions, consistent with the large number of assembly gaps. Nevertheless, the final annotation has a strong protein ratio peak around 1.0 for known proteins (Fig. 6b \& 6d), indicating that the bulk of these predicted genes were of lengths

830 similar to their Swiss-Prot homologs and hence deemed high quality. assemblies, with the resulting final gene count predictions comparable to other high-quality

834 versions reported similar final annotation statistics, with $S$. vulgaris vNA reporting slightly

835 more predicted genes (Table 3), and a larger predicted gene coverage over the genome

836 (59.09\% gene coverage vs $55.23 \%$ ), indicating this increase in predicted genes is not just a result of more overlapped predictions, though it could be a result of smaller assembly size and

838 higher gene duplication (Fig. 5a).

\section{Conclusion:}

842 the importance of diverse assessment tools in the assembly and assessment of reference

843 genomes and annotations. We present a high-quality, annotated S. vulgaris vAU reference

844 genome, scaffolded at the chromosome level. Alongside a second assembly, S. vulgaris vNA, 
these data provide vital resources for characterising the diverse and changing genomic

847 annotation, we demonstrate the utility of long-read transcript data for genome quality

848 assessment and assembly scaffolding. We also reveal some counter-intuitive behaviour of

849 BUSCO genome completeness statistics, and present complementary two tools, BUSCOMP

850 and SAAGA, which can identify and resolve potential artefacts, and inform assembly pipeline

851 decisions.

\section{Author Contributions}

853 Project conception: all authors

854 Sample Collection: KCS, SJW, MCB

855 Lab Work: KCS, YC, LAR, WCW

856 Data Analysis: KCS, RJE, YC, WCW

857 Program Development: RJE

858 Manuscript Writing: KCS, RJE

859 Manuscript Editing: All authors

\section{Acknowledgements:}

861 We thank non-author members of the Starling Genome Consortium for their support of this

862 project including Wim Vanden Berghe. Thank you to Stella Loke, Annabel Whibley, and

863 Mark Richardson for their guidance of Nanopore sequencing and analysis. Thank you to

864 Daniel Selechnik for assistance with RNA extractions. Art credit (Fig. 7 illustration) to

865 Megan Bishop. RJE was funded by the Australian Research Council (LP160100610 and

866 LP18010072). DWB and YC acknowledge grant funding from the Human Sciences Frontier

867 Programme (Grant RGP0030/2015). SM acknowledges Roslin Institute Strategic Grant

868 funding from the UK Biotechnology and Biological Sciences Research Council 
869 (BB/P013759/1). LAR was supported by a Scientia Fellowship from UNSW.

\section{Data Accessibility and Programs}

871 BUSCOMP documentation: https://slimsuite.github.io/buscomp/

872 Diploidocus documentation: https://slimsuite.github.io/diploidocus/

873 SAAGA documentation: https://slimsuite.github.io/saaga/

874 The data have been deposited with links to BioProject accession number PRJNA706841 in

875 the NCBI BioProject database (https://www.ncbi.nlm.nih.gov/bioproject/). Bioproject raw

876 data reviewer link is available at:

877 https://dataview.ncbi.nlm.nih.gov/object/PRJNA706841?reviewer=op1k3u6792jbg8o4rddq13

$878 \quad \underline{8 g} 9 \mathrm{e}$

879 Genome accession JAGFZL000000000 is currently private on NCBI, but a reviewer copy is

880 available here: https://drive.google.com/drive/folders/1MTExtlui-I-

881 ziCIzwyBtgiP0NwHTg3IG?usp=sharing

882 Any scripts or metadata not covered by the above will be available on GitHub.

883

884

885

886

887

888

889

890

891

892

893

894

895

896

897

898

899

900

901

\section{References:}

Backström N, Forstmeier W, Schielzeth H, Mellenius H, Nam K, Bolund E, Webster MT, Öst T, Schneider M, Kempenaers B et al. 2010 The recombination landscape of the zebra finch Taeniopygia guttata genome. Genome Research 20 485-495. (doi:10.1101/gr.101410.109)

Balakrishnan CN, Edwards SV \& Clayton DF 2010 The Zebra Finch genome and avian genomics in the wild. Emu - Austral Ornithology 110 233-241. (doi:10.1071/MU09087)

Bateson M \& Feenders G 2010 The use of passerine bird species in laboratory research: implications of basic biology for husbandry and welfare. ILAR Journal 51 394-408. (doi:10.1093/ilar.51.4.394)

BirdLife International 2020 Species factsheet: Sturnus vulgaris. In BirdLife International.

Bodt LH, Rollins LA \& Zichello J 2020 Genetic Diversity of the European Starling (Sturnus vulgaris) Compared Across Three Invasive Ranges.

Boetzer M \& Pirovano W 2014 SSPACE-LongRead: scaffolding bacterial draft genomes using long read sequence information. BMC Bioinformatics 15 211. (doi:10.1186/1471-2105-15-211)

Bomford M \& Sinclair R 2002 Australian research on bird pests: impact, management and future directions. Emu - Austral Ornithology 102 29-45. (doi:10.1071/MU01028)

Bradnam KR, Fass JN, Alexandrov A, Baranay P, Bechner M, Birol I, Boisvert S, Chapman JA, Chapuis G, Chikhi R et al. 2013 Assemblathon 2: evaluating de novo methods of genome assembly in three vertebrate species. GigaScience 2. (doi:10.1186/2047-217X-2-10) 
902

903

904

905

906

907

908

909

910

911

912

913

914

915

916

917

918

919

920

921

922

923

924

925

926

927

928

929

930

931

932

933

934

935

936

937

938

Calafati P \& Capanna E 1981 Karyotype analysis in ornithological studies: the chromosomes of six species of oscines (passeriformes). Avocetta 5 1-5.

Camacho C, Coulouris G, Avagyan V, Ma N, Papadopoulos J, Bealer K \& Madden TL 2009 BLAST+: architecture and applications. BMC Bioinformatics 10 421. (doi:10.1186/1471-2105-10-421)

Coleman JD 1977 The foods and feeding of starlings in Canterbury. Proceedings (New Zealand Ecological Society) 24 94-109.

Davey NE, Shields DC \& Edwards RJ 2006 SLiMDisc: short, linear motif discovery, correcting for common evolutionary descent. Nucleic Acids Research 34 3546-3554. (doi:10.1093/nar/gkl486)

Edwards R 2019 BUSCOMP: BUSCO compilation and comparison - Assessing completeness in multiple genome assemblies. F1000Research 8:995 (slides). (doi:10.7490/f1000research.1116972.1)

Edwards RJ, Tuipulotu DE, Amos TG, O'Meally D, Richardson MF, Russell TL, Vallinoto M, Carneiro M, Ferrand N, Wilkins MR et al. 2018 Draft genome assembly of the invasive cane toad, Rhinella marina. GigaScience 7. (doi:10.1093/gigascience/giy095)

Edwards RJ, Field MA, Ferguson JM, Dudchenko O, Keilwagen J, Rosen BD, Johnson GS, Rice ES, Hillier LD, Hammond JM et al. 2021 Chromosome-length genome assembly and structural variations of the primal Basenji dog (Canis lupus familiaris) genome. BMC Genomics 22188. (doi:10.1186/s12864-021-07493-6)

Feare CJ 1985 The Starling. Shire.

Field MA, Rosen BD, Dudchenko O, Chan EKF, Minoche AE, Edwards RJ, Barton K, Lyons RJ, Tuipulotu DE, Hayes VM et al. 2020 Canfam_GSD: De novo chromosome-length genome assembly of the German Shepherd Dog (Canis lupus familiaris) using a combination of long reads, optical mapping, and Hi-C. GigaScience 9. (doi:10.1093/gigascience/giaa027)

Flynn JM, Hubley R, Goubert C, Rosen J, Clark AG, Feschotte C \& Smit AF 2020 RepeatModeler2 for automated genomic discovery of transposable element families. Proceedings of the National Academy of Sciences 117 9451-9457. (doi:10.1073/pnas.1921046117)

Griffin DK, Robertson LBW, Tempest HG \& Skinner BM 2007 The evolution of the avian genome as revealed by comparative molecular cytogenetics. Cytogenetic and Genome Research 117 6477. (doi:10.1159/000103166)

Gs S \& E B 2005 Automated generation of heuristics for biological sequence comparison. BMC Bioinformatics 6 31-31. (doi:10.1186/1471-2105-6-31)

Gu Z, Gu L, Eils R, Schlesner M \& Brors B 2014 circlize Implements and enhances circular visualization in R. Bioinformatics (Oxford, England) 30 2811-2812. (doi:10.1093/bioinformatics/btu393)

Hirsch CN, Foerster JM, Johnson JM, Sekhon RS, Muttoni G, Vaillancourt B, Peñagaricano F, Lindquist E, Pedraza MA, Barry K et al. 2014 Insights into the Maize Pan-Genome and PanTranscriptome. The Plant Cell 26 121-135. (doi:10.1105/tpc.113.119982) 
Hofmeister NR, Werner SJ \& Lovette IJ 2019 Environment but not geography explains genetic variation in the invasive and largely panmictic European starling in North America. BioRxiv 643858. (doi:10.1101/643858)

Hofmeister NR, Stuart K, Warren WC, Werner SJ, Bateson M, Ball GF, Buchanan KL, Burt DW, Cardilini APA, Cassey P et al. 2021 Concurrent invasions by European starlings (Sturnus vulgaris) suggest selection on shared genomic regions even after genetic bottlenecks. BioRxiv 2021.05.19.442026. (doi:10.1101/2021.05.19.442026)

Holt C \& Yandell M 2011 MAKER2: an annotation pipeline and genome-database management tool for second-generation genome projects. BMC Bioinformatics 12 491. (doi:10.1186/14712105-12-491)

Hunt M, Kikuchi T, Sanders M, Newbold C, Berriman M \& Otto TD 2013 REAPR: a universal tool for genome assembly evaluation. Genome Biology 14 R47. (doi:10.1186/gb-2013-14-5-r47)

Hyatt D, Chen G-L, LoCascio PF, Land ML, Larimer FW \& Hauser L 2010 Prodigal: prokaryotic gene recognition and translation initiation site identification. BMC Bioinformatics 11119. (doi:10.1186/1471-2105-11-119)

Jayakumar V \& Sakakibara Y 2019 Comprehensive evaluation of non-hybrid genome assembly tools for third-generation PacBio long-read sequence data. Briefings in Bioinformatics 20 866-876. (doi:10.1093/bib/bbx147)

Keilwagen J, Hartung F, Paulini M, Twardziok SO \& Grau J 2018 Combining RNA-seq data and homology-based gene prediction for plants, animals and fungi. BMC Bioinformatics 19189. (doi:10.1186/s12859-018-2203-5)

Koch AJ, Munks SA \& Spencer C 2009 Bird use of native trees retained in young eucalypt plantations: species richness and use of hollows. Wildlife Research 36 581-591. (doi:10.1071/WR09037)

Kono N \& Arakawa K 2019 Nanopore sequencing: Review of potential applications in functional genomics. Development, Growth \& Differentiation 61 316-326. (doi:https://doi.org/10.1111/dgd.12608)

Korf I 2004 Gene finding in novel genomes. BMC Bioinformatics 5 59. (doi:10.1186/1471-2105-5-59)

Kuo RI, Cheng Y, Zhang R, Brown JWS, Smith J, Archibald AL \& Burt DW 2020 Illuminating the dark side of the human transcriptome with long read transcript sequencing. BMC Genomics 21 751. (doi:10.1186/s12864-020-07123-7)

Levy Karin E, Mirdita M \& Söding J 2020 MetaEuk—sensitive, high-throughput gene discovery, and annotation for large-scale eukaryotic metagenomics. Microbiome 8 48. (doi:10.1186/s40168020-00808-x)

Li H 2018 Minimap2: pairwise alignment for nucleotide sequences. Bioinformatics (Oxford, England) 34 3094-3100. (doi:10.1093/bioinformatics/bty191)

Linz GM, Homan HJ, Gaulker SM, Penry LB \& Bleier WJ 2007 European starlings: A review of an invasive species with far-reaching impacts. Managing Vertebrate Invasive Species. 
Linz G, Johnson R \& Thiele J 2017 European Starlings. In Ecology and Management of Terrestrial Vertebrate Invasive Species in the United States, 1st ed, pp 311-332. Ed WC Pitt. Boca Raton : Taylor \& Francis, 2018. | "A CRC title, part of the Taylor \& Francis imprint, a member of the Taylor \& Francis Group, the academic division of T\&F Informa plc.": CRC Press. (doi:10.1201/9781315157078-15)

Ma Z (Sam), Li L, Ye C, Peng M \& Zhang Y-P 2019 Hybrid assembly of ultra-long Nanopore reads augmented with 10x-Genomics contigs: Demonstrated with a human genome. Genomics 111 1896-1901. (doi:10.1016/j.ygeno.2018.12.013)

Mapleson D, Garcia Accinelli G, Kettleborough G, Wright J \& Clavijo BJ 2017 KAT: a K-mer analysis toolkit to quality control NGS datasets and genome assemblies. Bioinformatics 33 574-576. (doi:10.1093/bioinformatics/btw663)

Marçais G \& Kingsford C 2011 A fast, lock-free approach for efficient parallel counting of occurrences of k-mers. Bioinformatics 27 764-770. (doi:10.1093/bioinformatics/btr011)

Mirarab S, Nguyen N \& Warnow T 2012 SEPP: SATé-enabled phylogenetic placement. Pacific Symposium on Biocomputing. Pacific Symposium on Biocomputing 247-258. (doi:10.1142/9789814366496_0024)

O'Connor RE, Kiazim L, Skinner B, Fonseka G, Joseph S, Jennings R, Larkin DM \& Griffin DK 2019 Patterns of microchromosome organization remain highly conserved throughout avian evolution. Chromosoma 128 21-29. (doi:10.1007/s00412-018-0685-6)

Palacio FX, Maragliano RE \& Montalti D 2016 Functional role of the invasive European Starling, Sturnus vulgaris, in Argentina. Emu 116 387-393. (doi:10.1071/MU16021)

Peona V, Weissensteiner MH \& Suh A 2018 How complete are "complete" genome assemblies? -An avian perspective. Molecular Ecology Resources 18 1188-1195. (doi:https://doi.org/10.1111/1755-0998.12933)

Phair DJ, Roux JJL, Berthouly-Salazar C, Visser V, Vuuren BJ van, Cardilini APA \& Hui C 2018 Contextdependent spatial sorting of dispersal-related traits in the invasive starlings (Sturnus vulgaris) of South Africa and Australia. BioRxiv 342451. (doi:10.1101/342451)

Prentis PJ, Wilson JRU, Dormontt EE, Richardson DM \& Lowe AJ 2008 Adaptive evolution in invasive species. Trends in Plant Science 13 288-294. (doi:10.1016/j.tplants.2008.03.004)

Quinlan AR \& Hall IM 2010 BEDTools: a flexible suite of utilities for comparing genomic features. Bioinformatics 26 841-842. (doi:10.1093/bioinformatics/btq033)

Rhie A, McCarthy SA, Fedrigo O, Damas J, Formenti G, Koren S, Uliano-Silva M, Chow W, Fungtammasan A, Gedman GL et al. 2020 Towards complete and error-free genome assemblies of all vertebrate species. BioRxiv 2020.05.22.110833. (doi:10.1101/2020.05.22.110833)

Rice P, Longden I \& Bleasby A 2000 EMBOSS: the European Molecular Biology Open Software Suite. Trends in Genetics: TIG 16 276-277. (doi:10.1016/s0168-9525(00)02024-2) 
1013

1014

1015

1016

1017

1018

1019

1020

1021

1022

1023

1024

1025

1026

1027

1028

1029

1030

1031

1032

1033

1034

1035

1036

1037

1038

1039

1040

1041

1042

1043

1044

1045

1046

1047

1048

Richardson MF, Sherwin WB \& Rollins LA 2017 De Novo Assembly of the Liver Transcriptome of the European Starling, Sturnus vulgaris. Journal of Genomics 5 54-57. (doi:10.7150/jgen.19504)

Rintala J, Tiainen J \& Pakkala T 2003 Population trends of the Finnish starling Sturnus vulgaris, 19521998, as inferred from annual ringing totals. Annales Zoologici Fennici 40 365-385.

Robinson RA, Siriwardena GM \& Crick HQP 2005 Status and population trends of Starling Sturnus vulgaris in Great Britain. Bird Study 52 252-260. (doi:10.1080/00063650509461398)

Rosenberg KV, Dokter AM, Blancher PJ, Sauer JR, Smith AC, Smith PA, Stanton JC, Panjabi A, Helft L, Parr M et al. 2019 Decline of the North American avifauna. Science 366 120-124. (doi:10.1126/science.aaw1313)

Sherman RM \& Salzberg SL 2020 Pan-genomics in the human genome era. Nature Reviews Genetics 21 243-254. (doi:10.1038/s41576-020-0210-7)

Simão FA, Waterhouse RM, loannidis P, Kriventseva EV \& Zdobnov EM 2015 BUSCO: assessing genome assembly and annotation completeness with single-copy orthologs. Bioinformatics (Oxford, England) 31 3210-3212. (doi:10.1093/bioinformatics/btv351)

Smit A, Hubley R \& Green P 2013 RepeatMasker Open-4.0.

Spooner FEB, Pearson RG \& Freeman R 2018 Rapid warming is associated with population decline among terrestrial birds and mammals globally. Global Change Biology 24 4521-4531. (doi:https://doi.org/10.1111/gcb.14361)

Stanke M \& Morgenstern B 2005 AUGUSTUS: a web server for gene prediction in eukaryotes that allows user-defined constraints. Nucleic Acids Research 33 W465-W467. (doi:10.1093/nar/gki458)

Steinegger M \& Söding J 2017 MMseqs2 enables sensitive protein sequence searching for the analysis of massive data sets. Nature Biotechnology 35 1026-1028. (doi:10.1038/nbt.3988)

Stuart KC, Cardilini APA, Cassey P, Richardson MF, Sherwin W, Rollins LA \& Sherman CDH 2020 Signatures of selection in a recent invasion reveals adaptive divergence in a highly vagile invasive species. Molecular Ecology n/a. (doi:10.1111/mec.15601)

UniProt Consortium 2019 UniProt: a worldwide hub of protein knowledge. Nucleic Acids Research 47 D506-D515. (doi:10.1093/nar/gky1049)

Vernikos G, Medini D, Riley DR \& Tettelin H 2015 Ten years of pan-genome analyses. Current Opinion in Microbiology 23 148-154. (doi:10.1016/j.mib.2014.11.016)

Walker BJ, Abeel T, Shea T, Priest M, Abouelliel A, Sakthikumar S, Cuomo CA, Zeng Q, Wortman J, Young SK et al. 2014 Pilon: An Integrated Tool for Comprehensive Microbial Variant Detection and Genome Assembly Improvement. PLOS ONE 9 e112963. (doi:10.1371/journal.pone.0112963)

Weisenfeld NI, Kumar V, Shah P, Church DM \& Jaffe DB 2017 Direct determination of diploid genome sequences. Genome Research 27 757-767. (doi:10.1101/gr.214874.116) 
bioRxiv preprint doi: https://doi.org/10.1101/2021.04.07 438753. this version posted May 26. 2021. The copyriaht holder for this preprin (which was not certified by peer review) is the author/funder, who has granted bioRxiv a license to display the preprint in perpetuity. It is made available under aCC-BY-NC-ND 4.0 International license.

Wheeler TJ \& Eddy SR 2013 nhmmer: DNA homology search with profile HMMs. Bioinformatics 29 2487-2489. (doi:10.1093/bioinformatics/btt403)

Whibley A, Kelley JL \& Narum SR 2020 The changing face of genome assemblies: Guidance on achieving high-quality reference genomes. Molecular Ecology Resources. (doi:10.1111/17550998.13312)

Workman RE, Myrka AM, Wong GW, Tseng E, Welch KC \& Timp W 2018 Single-molecule, full-length transcript sequencing provides insight into the extreme metabolism of the ruby-throated hummingbird Archilochus colubris. GigaScience 7. (doi:10.1093/gigascience/giy009)

Wretenberg J, Lindström Å, Svensson S, Thierfelder T \& Pärt T 2006 Population trends of farmland birds in Sweden and England: similar trends but different patterns of agricultural intensification. Journal of Applied Ecology 43 1110-1120. (doi:10.1111/j.13652664.2006.01216.x)

Xue W, Li J-T, Zhu Y-P, Hou G-Y, Kong X-F, Kuang Y-Y \& Sun X-W 2013 L_RNA_scaffolder: scaffolding genomes with transcripts. BMC Genomics 14 604. (doi:10.1186/1471-2164-14-604)

Ye J, Zhang Y, Cui H, Liu J, Wu Y, Cheng Y, Xu H, Huang X, Li S, Zhou A et al. 2018 WEGO 2.0: a web tool for analyzing and plotting $\mathrm{GO}$ annotations, 2018 update. Nucleic Acids Research 46 W71-W75. (doi:10.1093/nar/gky400)

Yin Z, Zhang F, Smith J, Kuo R \& Hou Z-C 2019 Full-length transcriptome sequencing from multiple tissues of duck, Anas platyrhynchos. Scientific Data 6 275. (doi:10.1038/s41597-019-0293-1)

Yuan Y, Bayer PE, Scheben A, Chan C-KK \& Edwards D 2017 BioNanoAnalyst: a visualisation tool to assess genome assembly quality using BioNano data. BMC Bioinformatics 18323. (doi:10.1186/s12859-017-1735-4) 